\title{
Measurement of cross sections
}

\section{and leptonic forward-backward asymmetries at the $Z$ pole and determination of electroweak parameters}

\section{L3 Collaboration}

M. Acciarri ${ }^{25}$ A. Adam ${ }^{43}$, O. Adriani ${ }^{15}$, M. Aguilar-Benitez ${ }^{24}$, S. Ahlen ${ }^{9}$, J. Alcaraz ${ }^{16}$, A. Aloisio ${ }^{27}$, G. Alverson ${ }^{10}$, M.G. Alviggi $^{27}$, G. Ambrosi ${ }^{32}$, Q. An ${ }^{17}$, H. Anderhub ${ }^{46}$, A.L. Anderson ${ }^{14}$, V.P. Andreev ${ }^{36}$, T. Angelescu ${ }^{11}$, L. Antonov ${ }^{40}$, D. Antreasyan $^{7}$, G. Alkhazov ${ }^{36}$, P. Arce ${ }^{24}$, A. Arefiev ${ }^{26}$, T. Azemoon ${ }^{3}$, T. Aziz ${ }^{8}$, P.V.K.S. Baba ${ }^{17}$, P. Bagnaia ${ }^{35}$, J.A. Bakken ${ }^{34}$, L. Baksay ${ }^{42}$, R.C. Ball ${ }^{3}$, S. Banerjee ${ }^{8}$, K. Banicz ${ }^{43}$, J. Bao ${ }^{5}$, R. Barillère ${ }^{16}$, L. Barone ${ }^{35}$, A. Baschirotto ${ }^{25}$, R. Battiston ${ }^{32}$, A. Bay $^{18}$, F. Becattini ${ }^{15}$, U. Becker ${ }^{14}$, F. Behner ${ }^{46}$, Gy.L. Bencze ${ }^{12}$, J. Berdugo ${ }^{24}$, P. Berges ${ }^{14}$, B. Bertucci ${ }^{32}$, B.L. Betev ${ }^{40,46}$, M. Biasini ${ }^{32}$, A. Biland ${ }^{46}$, G.M. Bilei ${ }^{32}$, R. Bizzarri ${ }^{35}$, J.J. Blaising ${ }^{4}$, G.J. Bobbink ${ }^{16,2}$, R. Bock ${ }^{1}$, A. Böhm ${ }^{1}$, B. Borgia ${ }^{35}$, D. Bourilkov $^{46}$, M. Bourquin ${ }^{18}$, D. Boutigny ${ }^{16}$, B. Bouwens ${ }^{2}$, E. Brambilla ${ }^{27}$, J.G. Branson ${ }^{37}$, V. Brigljevic ${ }^{46}$, I.C. Brock ${ }^{33}$, M. Brooks ${ }^{22}$, A. Bujak ${ }^{43}$, J.D. Burger ${ }^{14}$, W.J. Burger ${ }^{18}$, C. Burgos ${ }^{24}$, J. Busenitz ${ }^{42}$, A. Buytenhuijs ${ }^{29}$, A. Bykov ${ }^{36}$, X.D. $\mathrm{Cai}^{17}$, M. Capell ${ }^{14}$, M. Caria ${ }^{32}$, G. Carlino ${ }^{27}$, A.M. Cartacci ${ }^{15}$, J. Casaus ${ }^{24}$, R. Castello ${ }^{25}$, N. Cavallo ${ }^{27}$, M. Cerrada ${ }^{24}$, F. Cesaroni $^{35}$, M. Chamizo ${ }^{24}$, Y.H. Chang ${ }^{48}$, U.K. Chaturvedi ${ }^{17}$, M. Chemarin ${ }^{23}$, A. Chen ${ }^{48}$, C. Chen ${ }^{6}$, G. Chen ${ }^{6,46}$, G.M. Chen $^{6}$, H.F. Chen ${ }^{19}$, H.S. Chen ${ }^{6}$, M. Chen ${ }^{14}$, G. Chiefari ${ }^{27}$, C.Y. Chien ${ }^{5}$, M.T. Choi ${ }^{41}$, S. Chung ${ }^{14}$, C. Civinini ${ }^{15}$, I. Clare ${ }^{14}$, R. Clare ${ }^{14}$, T.E. Coan $^{22}$, H.O. Cohn ${ }^{30}$, G. Coignet $^{4}$, N. Colino ${ }^{16}$, A. Contin ${ }^{7}$, S. Costantini ${ }^{35}$, F. Cotorobai ${ }^{11}$, B. de la Cruz ${ }^{24}$, X.T. Cui ${ }^{17}$, X.Y. Cui ${ }^{17}$, T.S. Dai ${ }^{14}$, R. D'Alessandro ${ }^{15}$, R. de Asmundis ${ }^{27}$, A. Degré ${ }^{4}$, K. Deiters ${ }^{44}$, E. Dénes ${ }^{12}$, P. Denes ${ }^{34}$, F. DeNotaristefani ${ }^{35}$, D. DiBitonto ${ }^{42}$, M. Diemoz ${ }^{35}$, H.R. Dimitrov ${ }^{40}$, C. Dionisi ${ }^{35}$, M. Dittmar ${ }^{46}$, L. Djambazov ${ }^{46}$, M.T. Dova $^{17 a}$, E. Drago ${ }^{27}$, D. Duchesneau ${ }^{18}$, P. Duinker ${ }^{2}$, I. Duran ${ }^{38}$, S. Easo ${ }^{32}$, H. El Mamouni ${ }^{23}$, A. Engler ${ }^{33}$, F.J. Eppling ${ }^{14}$, F.C. Erné ${ }^{2}$, P. Extermann ${ }^{18}$, R. Fabbretti ${ }^{44}$, M. Fabre ${ }^{44}$, S. Falciano ${ }^{35}$, S.J. Fan ${ }^{39}$, A. Favara ${ }^{15}$, J. Fay ${ }^{23}$, M. Felcini ${ }^{46}$, T. Ferguson $^{33}$, D. Fernandez ${ }^{24}$, G. Fernandez ${ }^{24}$, F. Ferroni ${ }^{35}$, H. Fesefeldt ${ }^{1}$, E. Fiandrini ${ }^{32}$, J.H. Field ${ }^{18}$, F. Filthaut ${ }^{29}$, P.H. Fisher $^{5}$, G. Forconi ${ }^{14}$, L. Fredj ${ }^{18}$, K. Freudenreich ${ }^{46}$, W. Friebel ${ }^{45}$, M. Fukushima ${ }^{14}$, M. Gailloud ${ }^{21}$, Yu. Galaktionov ${ }^{26,14}$, E. Gallo ${ }^{15}$, S.N. Ganguli ${ }^{8}$, P. Garcia-Abia ${ }^{24}$, S. Gentile ${ }^{35}$, N. Gheordanescu ${ }^{11}$, S. Giagu ${ }^{35}$, S. Goldfarb ${ }^{21}$, Z.F. Gong ${ }^{19}$, E. Gonzalez $^{24}$, A. Gougas ${ }^{5}$, D. Goujon ${ }^{18}$, G. Gratta ${ }^{31}$, M.W. Gruenewald ${ }^{16}$, C. Gu ${ }^{17}$, M. Guanziroli ${ }^{17}$, J.K. Guo ${ }^{39}$, V.K. Gupta ${ }^{34}$, A. Gurtu ${ }^{8}$, H.R. Gustafson ${ }^{3}$, L.J. Gutay ${ }^{43}$, K. Hangarter ${ }^{1}$, A. Hasan ${ }^{17}$, D. Hauschildt ${ }^{2}$, C.F. He ${ }^{39}$, J.T. He ${ }^{6}$, T. Hebbeker ${ }^{16}$, M. Hebert ${ }^{37}$, A. Hervé ${ }^{16}$, K. Hilgers ${ }^{1}$, H. Hofer ${ }^{46}$, H. Hoorani ${ }^{18}$, S.R. Hou ${ }^{48}$, G. Hu ${ }^{17}$, G.Q. Hu ${ }^{39}$, B. Ille ${ }^{23}$, M.M. Ilyas ${ }^{17}$, V. Innocente ${ }^{16}$, H. Janssen ${ }^{4}$, B.N. Jin ${ }^{6}$, L.W. Jones ${ }^{3}$, I. Josa-Mutuberria ${ }^{16}$, A. Kasser ${ }^{21}$, R.A. Khan ${ }^{17}$, Yu. Kamyshkov ${ }^{30}$, P. Kapinos $^{45}$, J.S. Kapustinsky ${ }^{22}$, Y. Karyotakis ${ }^{16}$, M. Kaur ${ }^{17}$, S. Khokhar ${ }^{17}$, M.N. Kienzle-Focacci ${ }^{18}$, J.K. Kim ${ }^{41}$, S.C. Kim ${ }^{41}$, Y.G. Kim ${ }^{41}$, W.W. Kinnison ${ }^{22}$, A. Kirkby ${ }^{31}$, D. Kirkby ${ }^{31}$, S. Kirsch ${ }^{45}$, W. Kittel ${ }^{29}$, A. Klimentov ${ }^{14,26}$, A.C. König ${ }^{29}$, E. Koffeman $^{2}$, O. Kornadt ${ }^{1}$, V. Koutsenko ${ }^{14,26}$, A. Koulbardis ${ }^{36}$, R.W. Kraemer ${ }^{33}$, T. Kramer ${ }^{14}$, V.R. Krastev ${ }^{40,32}$, W. Krenz ${ }^{1}$, H. Kuijten ${ }^{29}$, K.S. Kumar ${ }^{13}$, A. Kunin ${ }^{14,26}$, P. Ladron de Guevara ${ }^{24}$, G. Landi ${ }^{15}$, D. Lanske ${ }^{1}$, S. Lanzano ${ }^{27}$, A. Lebedev ${ }^{14}$, P. Lebrun ${ }^{23}$, P. Lecomte ${ }^{46}$, P. Lecoq ${ }^{16}$, P. Le Coultre ${ }^{46}$, D.M. Lee ${ }^{22}$, J.S. Lee ${ }^{41}$, K.Y. Lee ${ }^{41}$, I. Leedom ${ }^{10}$, C. Leggett ${ }^{3}$, J.M. Le Goff ${ }^{16}$, R. Leiste ${ }^{45}$, M. Lenti ${ }^{15}$, E. Leonardi ${ }^{35}$, P. Levtchenko ${ }^{36}$, C. Li ${ }^{19}, 17$, H.T. Li ${ }^{6}$, P.J. Li ${ }^{39}$, J.Y. Liao ${ }^{39}$, W.T. Lin $^{48}$, Z.Y. Lin ${ }^{19}$, F.L. Linde ${ }^{2}$, B. Lindemann ${ }^{1}$, L. Lista ${ }^{27}$, Y. Liu ${ }^{17}$, W. Lohmann ${ }^{45}$, E. Longo ${ }^{35}$, W. Lu ${ }^{31}$, Y.S. Lu', J.M. Lubbers $^{16}$, K. Lübelsmeyer ${ }^{1}$, C. Luci ${ }^{35}$, D. Luckey ${ }^{7}{ }^{14}$, L. Ludovici ${ }^{35}$, L. Luminari ${ }^{35}$, W. Lustermann ${ }^{44}$, J.M. Ma ${ }^{6}$, W.G. $\mathrm{Ma}^{19}$, M. MacDermott ${ }^{46}$, L. Malgeri ${ }^{35}$, R. Malik ${ }^{17}$, A. Malinin ${ }^{26}$, C. Maña ${ }^{24}$, M. Maolinbay ${ }^{46}$, P. Marchesini ${ }^{46}$, F. Marion ${ }^{4}$, A. Marin 9 , J.P. Martin ${ }^{23}$, F. Marzano ${ }^{35}$, G.G.G. Massaro ${ }^{2}$, K. Mazumdar ${ }^{8}$, P. McBride ${ }^{13}$, T. McMahon ${ }^{43}$, D. McNally ${ }^{37}$, M. Merk ${ }^{33}$, L. Merola ${ }^{27}$, M. Meschini15, W.J. Metzger ${ }^{29}$, Y. Mi ${ }^{21}$, A. Mihul ${ }^{11}$, G.B. Mills ${ }^{22}$, Y. Mir ${ }^{17}$, G. Mirabelli ${ }^{35}$, J. Mnich $^{1}$, M. Möller ${ }^{1}$, B. Monteleoni ${ }^{15}$, R. Morand ${ }^{4}$, S. Morganti' ${ }^{35}$, N.E. Moulai ${ }^{17}$, R. Mount ${ }^{31}$, S. Müller ${ }^{1}$, E. Nagy ${ }^{12}$, M. Napolitano $^{27}$, F. Nessi-Tedaldi ${ }^{46}$, H. Newman ${ }^{31}$, M.A. Niaz ${ }^{17}$, A. Nippe ${ }^{1}$, H. Nowak ${ }^{45}$, G. Organtini ${ }^{35}$, D. Pandoulas ${ }^{1}$, S. Paoletti $^{35}$, P. Paolucci ${ }^{27}$, G. Pascale ${ }^{35}$, G. Passaleva ${ }^{15,32}$, S. Patricelli ${ }^{27}$, T. Paul ${ }^{5}$, M. Pauluzzi ${ }^{32}$, C. Paus ${ }^{1}$, F. Pauss ${ }^{46}$, Y.J. Pei $^{1}$, S. Pensotti ${ }^{25}$, D. Perret-Gallix ${ }^{4}$, J. Perrier ${ }^{18}$, A. Pevsner ${ }^{5}$, D. Piccolo ${ }^{27}$, M. Pieri ${ }^{16}$, J.C. Pinto ${ }^{33}$, P.A. Piroué ${ }^{34}$, F. Plasil $^{30}$, V. Plyaskin ${ }^{26}$, M. Pohl ${ }^{46}$, V. Pojidaev ${ }^{26,15}$, H. Postema ${ }^{14}$, Z.D. Qi ${ }^{39}$, J.M. Qian ${ }^{3}$, K.N. Qureshi ${ }^{17}$, R. Raghavan ${ }^{8}$, G. Rahal-Callot ${ }^{46}$, P.G. Rancoita ${ }^{25}$, M. Rattaggi ${ }^{25}$, G. Raven ${ }^{2}$, P. Razis ${ }^{28}$, K. Read ${ }^{30}$, M. Redaelli ${ }^{25}$, D. Ren ${ }^{46}, Z^{2}$ Ren ${ }^{17}$, M. Rescigno ${ }^{35}$, S. Reucroft ${ }^{10}$, A. Ricker ${ }^{1}$, S. Riemann ${ }^{45}$, B.C. Riemers ${ }^{43}$, K. Riles ${ }^{3}$, O. Rind ${ }^{3}$, H.A. Rizvi ${ }^{17}$, S. Ro ${ }^{41}$, A. Robohm ${ }^{46}$, F.J. Rodriguez ${ }^{24}$, B.P. Roe ${ }^{3}$, M. Röhner ${ }^{1}$, S. Röhner ${ }^{1}$, L. Romero ${ }^{24}$, S. Rosier-Lees ${ }^{4}$, R. Rosmalen ${ }^{29}$, Ph. Rosselet $^{21}$, W. van Rossum ${ }^{2}$, S. Roth ${ }^{1}$, A. Rubbia ${ }^{14}$, J.A. Rubio ${ }^{16}$, H. Rykaczewski ${ }^{46}$, M. Sachwitz ${ }^{45}$, J. Salicio ${ }^{16}$, J.M. Salicio $^{24}$, E. Sanchez ${ }^{24}$, G.S. Sanders ${ }^{22}$, A. Santocchia ${ }^{32}$, M.S. Sarakinos ${ }^{14}$, G. Sartorelli ${ }^{7,17}$, M. Sassowsky ${ }^{1}$, G. Sauvage ${ }^{4}$, C. Schäfer ${ }^{1}$, V. Schegelsky ${ }^{36}$, D. Schmitz ${ }^{1}$, P. Schmitz ${ }^{1}$, M. Schneegans ${ }^{4}$, N. Scholz ${ }^{46}$, H. Schopper ${ }^{47}$, D.J. Schotanus ${ }^{29}$, S. Shotkin $^{14}$, H.J. Schreiber ${ }^{45}$, J. Shukla ${ }^{33}$, R. Schulte ${ }^{1}$, K. Schultze ${ }^{1}$, J. Schwenke ${ }^{1}$, G. Schwering ${ }^{1}$, C. Sciacca ${ }^{27}$, I. Scott ${ }^{13}$, R. Sehgal ${ }^{17}$, P.G. Seiler ${ }^{44}$, J.C. Sens ${ }^{16,2}$, L. Servoli ${ }^{32}$, I. Sheer ${ }^{37}$, D.Z. Shen ${ }^{39}$, S. Shevchenko ${ }^{31}$, X.R. Shi ${ }^{31}$, E. Shumilov ${ }^{26}$, V. Shoutko ${ }^{26}$, D. Son ${ }^{41}$, A. Sopczak ${ }^{16}$, V. Soulimov ${ }^{27}$, C. Spartiotis ${ }^{20}$, T. Spickermann ${ }^{1}$, P. Spillantini ${ }^{15}$, R. Starosta ${ }^{1}$, M. Steuer ${ }^{7,14}$, D.P. Stickland ${ }^{34}$, F. Sticozzi ${ }^{14}$, H. Stone ${ }^{34}$, K. Strauch ${ }^{13}$, K. Sudhakar ${ }^{8}$, G. Sultanov ${ }^{17}$, L.Z. Sun ${ }^{19,17}$, G.F. Susinno $^{18}$, H. Suter ${ }^{46}$, J.D. Swain ${ }^{17}$, A.A. Syed ${ }^{29}$, X.W. Tang ${ }^{6}$, L. Taylor ${ }^{10}$, S.C.C. Ting ${ }^{14}$, S.M. Ting ${ }^{14}$, O. Toker ${ }^{32}$, M.

a Also supported by CONICET and Universidad Nacional de La Plata, CC 67, 1900 La Plata, Argentina 
Tonutti $^{1}$, S.C. Tonwar ${ }^{8}$, J. Tóth ${ }^{12}$, A. Tsaregorodtsev ${ }^{36}$, G. Tsipolitis ${ }^{33}$, C. Tully ${ }^{34}$, K.L. Tung ${ }^{6}$, T. Tuuva ${ }^{20}$, J. Ulbricht ${ }^{46}$, L. Urbán $^{12}$, U. Uwer ${ }^{1}$, E. Valente ${ }^{35}$, R.T. Van de Walle ${ }^{29}$, I. Vetlitsky ${ }^{26}$, G. Viertel ${ }^{46}$, P. Vikas ${ }^{17}$, U. Vikas ${ }^{17}$, M. Vivargent ${ }^{4}, H$ Vogel $^{33}$, H. Vogt ${ }^{45}$, I. Vorobiev ${ }^{13,26}$, A.A. Vorobyov ${ }^{36}$, An.A. Vorobyov ${ }^{36}$, L. Vuilleumier ${ }^{21}$, M. Wadhwa ${ }^{4}$, W. Wallraff ${ }^{1}$, J.C. Wang $^{14}$, C.R. Wang ${ }^{19}$, X.L. Wang ${ }^{19}$, Y.F. Wang ${ }^{14}$, Z.M. Wang ${ }^{17,19}$, A. Weber ${ }^{1}$, J. Weber ${ }^{46}$, R. Weill ${ }^{21}$, J. Wenninger ${ }^{18}$, M. White $^{14}$, C. Willmott ${ }^{24}$, F. Wittgenstein ${ }^{16}$, D. Wright ${ }^{34}$, S.X. Wu ${ }^{17}$, S. Wynhoff ${ }^{1}$, B. Wyslouch ${ }^{14}$, Y.Y. Xie ${ }^{39}$, J.G. Xu ${ }^{6}, Z . Z$. $\mathrm{Xu}^{19}$, Z.L. Xue ${ }^{39}$, D.S. Yan $^{39}$, B.Z. Yang ${ }^{19}$, C.G. Yang ${ }^{6}$, G. Yang ${ }^{17}$, C.H. Ye ${ }^{17}$, J.B. Ye ${ }^{19}$, Q. Ye ${ }^{17}$, S.C. Yeh ${ }^{48}$, Z.W. Yin ${ }^{39}$, J.M. You ${ }^{17}$, N. Yunus ${ }^{17}$, M. Yzerman ${ }^{2}$, C. Zaccardelli ${ }^{31}$, P. Zemp ${ }^{46}$, M. Zeng ${ }^{17}$, Y. Zeng ${ }^{1}$, D.H. Zhang ${ }^{2}$, Z.P. Zhang ${ }^{19,17}$, B. Zhou $^{9}$, G.J. Zhou', J.F. Zhou', R.Y. Zhu' ${ }^{31}$, A. Zichichi' ${ }^{7}{ }^{76,17}$, and B.C.C. van der Zwaan ${ }^{2}$

1 I. Physikalisches Institut, III. Physikalisches Institut, RWTH, D-52056 Aachen, Germany ${ }^{b}$

2 National Institute for High Energy Physics, NIKHEF, NL-1009 DB Amsterdam, The Netherlands

3 University of Michigan, Ann Arbor, MI 48109, USA

${ }^{4}$ Laboratoire d'Annecy-le-Vieux de Physique des Particules, LAPP,IN2P3-CNRS, BP 110, F-74941 Annecy-le-Vieux Cedex, France

5 Johns Hopkins University, Baltimore, MD 21218, USA

6 Institute of High Energy Physics, IHEP, 100039 Beijing, People's Republic of China

7 INFN-Sezione di Bologna, I-40126 Bologna, Italy

8 Tata Institute of Fundamental Research, Bombay 400 005, India

9 Boston University, Boston, MA 02215, USA

${ }^{10}$ Northeastern University, Boston, MA 02115, USA

11 . Institute of Atomic Physics and University of Bucharest, RO-76900 Bucharest, Romania

12 Central Research Institute for Physics of the Hungarian Academy of Sciences, H-1525 Budapest 114, Hungary ${ }^{c}$

13 Harvard University, Cambridge, MA 02139, USA

14 Massachusetts Institute of Technology, Cambridge, MA 02139, USA

15 INFN Sezione di Firenze and University of Florence, I-50125 Florence, Italy

16 European Laboratory for Particle Physics, CERN, CH-1211 Geneva 23, Switzerland

17 World Laboratory, FBLJA Project, CH-1211 Geneva 23, Switzerland

18 University of Geneva, CH-1211 Geneva 4, Switzerland

19 Chinese University of Science and Technology, USTC, Hefei, Anhui 230 029, People's Republic of China

20 SEFT, Research Institute for High Energy Physics, P.O. Box 9, FIN-00014 Helsinki, Finland

21 University of Lausanne, $\mathrm{CH}-1015$ Lausanne, Switzerland

22 Los Alamos National Laboratory, Los Alamos, NM 87544, USA

23 Institut de Physique Nucléaire de Lyon, IN2P3-CNRS,Université Claude Bernard, F-69622 Villeurbanne Cedex, France

24 Centro de Investigaciones Energeticas, Medioambientales y Tecnologicas, CIEMAT, E-28040 Madrid, Spain

25 INFN-Sezione di Milano, I-20133 Milan, Italy

26 Institute of Theoretical and Experimental Physics, ITEP, Moscow, Russia

27 INFN-Sezione di Napoli and University of Naples, I-80125 Naples, Italy

28 Department of Natural Sciences, University of Cyprus, Nicosia, Cyprus

29 University of Nijmegen and NIKHEF, NL-6525 ED Nijmegen, The Netherlands

30) Oak Ridge National Laboratory, Oak Ridge, TN 37831, USA

31 California Institute of Technology, Pasadena, CA 91125, USA

32 INFN-Sezione di Perugia and Universitá Degli Studi di Perugia, I-06100 Perugia, Italy

33 Carnegie Mellon University, Pittsburgh, PA 15213, USA

34 Princeton University, Princeton, NJ 08544, USA

35 INFN-Sezione di Roma and University of Rome, "La Sapienza", I-00185 Rome, Italy

36 Nuclear Physics Institute, St. Petersburg, Russia

37 University of California, San Diego, CA 92093, USA

38 Dept. de Fisica de Particulas Elementales, Univ. de Santiago, E-15706 Santiago de Compostela, Spain

39 Shanghai Institute of Ceramics, SIC, Shanghai, China

40) Bulgarian Academy of Sciences, Institute of Mechatronics, BU-1113 Sofia, Bulgaria

41 Center for High Energy Physics, Korea Advanced Inst. of Sciences and Technology, 305-701 Taejon, Republic of Korea

42 University of Alabama, Tuscaloosa, AL 35486, USA

43 Purdue University, West Lafayette, IN 47907, USA

44 Paul Scherrer Institut, PSI, CH-5232 Villigen, Switzerland

45 DESY-Institut für Hochenergiephysik, D-15738 Zeuthen, Germany

46 Eidgenössische Technische Hochschule, ETH Zürich, CH-8093 Zürich, Switzerland

47 University of Hamburg, D-22761 Hamburg, Germany

48 High Energy Physics Group, Taiwan, China

Received: 15 March 1994

\footnotetext{
${ }^{b}$ Supported by the German Bundesministerium für Forschung und Technologie

c Supported by the Hungarian OTKA fund under contract number 2970
}

Abstract. We report on the measurement of the leptonic and hadronic cross sections and leptonic forward-backward asymmetries at the $Z$ peak with the L3 detector at LEP. The total luminosity of $40.8 \mathrm{pb}^{-1}$ collected in the years 1990,1991 and 1992 corresponds to $1.09 \cdot 10^{6}$ hadronic and $0.98 \cdot 10^{5}$ leptonic $Z$ decays observed. These data allow us to determine the electroweak parameters. From the cross sections we derive the properties of the $Z$ boson: 
$M_{\mathrm{Z}}=91195 \pm 9 \mathrm{MeV} \quad \Gamma_{\mathrm{Z}}=2494 \pm 10 \mathrm{MeV}$

$\Gamma_{\text {had }}=1748 \pm 10 \mathrm{MeV} \Gamma_{\ell}=83.49 \pm 0.46 \mathrm{MeV}$,

assuming lepton universality. We obtain an invisible width of $\Gamma_{\mathrm{inv}}=496.5 \pm 7.9 \mathrm{MeV}$ which, in the Standard Model, corresponds to a number of light neutrino species of $\mathrm{N}_{\nu}=$ $2.981 \pm 0.050$.

Using also the three leptonic forward-backward asymmetries and the average tau polarization, we determine the effective vector and axial-vector coupling constants of the neutral weak current to charged leptons to be:

$\bar{g}_{V}^{\ell}=-0.0378_{-0.0042}^{+0.0045} \quad \bar{g}_{A}^{\ell}=-0.4998 \pm 0.0014$.

Within the framework of the Standard Model, and including our measurements of the $\mathrm{Z} \rightarrow \mathrm{b} \overline{\mathrm{b}}$ forward-backward asymmetry and partial decay width, we derive an effective electroweak mixing angle of $\sin ^{2} \bar{\theta}_{W}=0.2326 \pm 0.0012$. We obtain an estimate for the strong coupling constant, $\alpha_{\mathrm{s}}=0.142 \pm 0.013$, and for the top-quark mass, $m_{t}=$ $158_{-40}^{+32} \pm 19$ (Higgs) $\mathrm{GeV}$, where the second error arises due to the uncertainty in the Higgs-boson mass.

\section{Introduction}

In 1992, the LEP $e^{+} e^{-}$collider at CERN ran at the centerof-mass energy $91.29 \mathrm{GeV}$, where the cross section for $Z$ boson production in $e^{+} e^{-}$annihilation is maximum. A total luminosity of $22.4 \mathrm{pb}^{-1}$ was recorded with the L3 detector at LEP corresponding to 678,000 hadronic and 59,000 leptonic $Z$ decays selected.

In this article we report on the measurements of the reactions:
1. $e^{+} e^{-} \rightarrow$ hadrons $(\gamma)$,
2. $e^{+} e^{-} \rightarrow \mu^{+} \mu^{-}(\gamma)$,
3. $e^{+} e^{-} \rightarrow \tau^{+} \tau^{-}(\gamma)$,
4. $e^{+} e^{-} \rightarrow e^{+} e^{-}(\gamma)$,

where the $(\gamma)$ indicates the presence of radiative photons. These measurements are used to determine the parameters of the Standard Model (SM) $[1,2]$.

Earlier measurements of the reactions $1-4$ have been reported by the LEP experiments [3-5]. The inclusion of the 1992 data doubles the peak event sample. Detailed studies are performed with this large sample of events with the aim of decreasing the systematic errors. These studies allow us to exploit the full statistical accuracy of our data.

Since the data analysis is improved compared to our earlier work of the years 1990 and 1991 [3, 4], we also reanalyze that data. Here we present the results derived from the data collected by L3 in the years 1990, 1991 and 1992, which correspond to a total luminosity of $40.8 \mathrm{pb}^{-1}$, or $1.09 \cdot 10^{6}$ hadronic and $0.98 \cdot 10^{5}$ leptonic $Z$ decays observed.

The organization of this article is: in Sect. 2 we briefly describe the L3 detector. The measurement of luminosity is discussed in Sect. 3. Section 4 presents the analysis of the reactions $1-4$. Our results on the leptonic and hadronic cross sections and leptonic forward-backward asymmetries are given in Sect. 5. The determination of the electroweak parameters is described in Sect. 6. A summary and conclusion are given in Sect. 7.

\section{The $\mathrm{L3}$ detector}

The L3 detector [6] is designed to measure the energy and direction of leptons, photons and jets with high precision. The detector consists of a central tracking chamber (TEC), a high resolution electromagnetic calorimeter composed of bismuth germanate crystals (BGO), a cylindrical array of scintillation counters (SCNT), a uranium and brass hadron calorimeter with proportional wire chamber readout (HCAL), and a precise muon spectrometer (MUCH). All elements are installed inside a magnet of $12 \mathrm{~m}$ diameter, which provides a uniform field of $0.5 \mathrm{~T}$ along the beam direction.

In the $\mathrm{L} 3$ detector $e^{+} e^{-}$interactions are recorded based on several independent triggers [6]. The trigger decisions are derived from the energy depositions in the calorimeters, tracks in the central tracking chamber or the muon spectrometer, or from multiple hits in the scintillation counters. The decays of the $Z$ into hadrons or charged leptons usually fulfill at least two trigger requirements which allows us to determine the individual and combined trigger efficiencies.

The response of the L3 detector is modeled with the GEANT 3.15 [7] detector simulation program which includes the effects of energy loss, multiple scattering and showering in the detector materials and in the beam pipe. Hadronic showers are simulated with the GHEISHA 8 [8] program.

\section{Measurement of luminosity}

The total luminosity, $\mathscr{B}$, is determined by measuring the number of small-angle Bhabha scatterings, $e^{+} e^{-} \rightarrow e^{+} e^{-}(\gamma)$. For this purpose two cylindrical calorimeters with an inner radius of $68.2 \mathrm{~mm}$ and an outer radius of $191.4 \mathrm{~mm}$ are located on either side of the interaction point at $z= \pm 2.7 \mathrm{~m}$. Each is a finely segmented and azimuthally symmetric array of 304 BGO crystals which is split in the vertical plane.

Event selection. The Bhabha event selection is based on the energy depositions in adjacent crystals of the two calorimeters which are grouped to form clusters. The selection criteria are:

1. One cluster is required to have the reconstructed radial $(R)$ and azimuthal $(\phi)$ impact coordinates, determined in the plane transverse to the beam, more than one crystal size away from the calorimeter edges:

a) $84.4<R<176.2 \mathrm{~mm}$

b) $\left|\phi-90^{\circ}\right|>11.25^{\circ}$ and $\left|\phi-270^{\circ}\right|>11.25^{\circ}$.

We impose no restrictions on the reconstructed impact coordinates on the opposite side.

2. The reconstructed energy on one side, $E_{\max }$, must be greater than $0.8 E_{\text {beam }}$, and that on the other side must be greater than $0.4 E_{\text {beam }}$, where $E_{\text {beam }}$ is the energy of the $e^{+} e^{-}$ beams in LEP. 

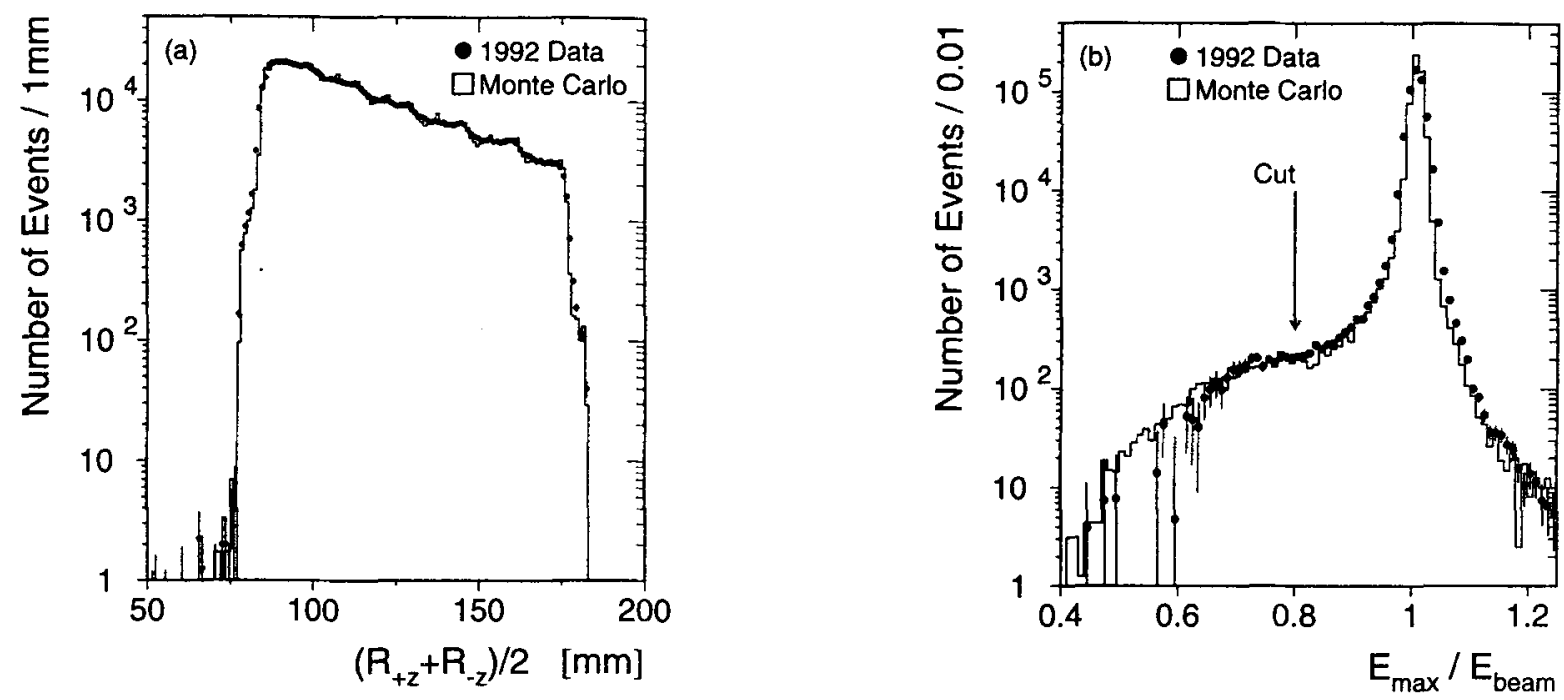

Fig. 1. a,b Distributions of variables used for the selection of luminosity $e^{+} e^{-} \rightarrow e^{+} e^{-}(\gamma)$ events, comparing the 1992 data to the simulated Monte-Carlo Bhabha events. Only the statistical errors on the data are shown. The statistical errors on the Monte-Carlo simulation are approximately twice as large. a The average reconstructed radial impact coordinate, $\left(R_{+z}+R_{-z}\right) / 2$. The wiggles in the distributions are due to the changing angular resolution across the face of each crystal. $\mathbf{b}$ The maximum of the reconstructed energies in the two luminosity calorimeters, $E_{\max }$, normalized to the beam energy, $E_{\text {beam }}$. The position of the selection cut is indicated by the vertical arrow. For energies below the cut, the errors on the data points are large, because the background, which has been subtracted, is significant

3. The coplanarity angle between the two clusters, $\Delta \phi$, corrected for the bending of the particle trajectory in the magnetic field, must satisfy $\left|\Delta \phi-180^{\circ}\right|<10^{\circ}$.

Figure 1 shows the distributions of the average radial impact coordinate, $R$, and the maximum energy, $E_{\max }$, comparing the data to fully simulated Monte-Carlo events.

Two samples of Bhabha events are maintained. In the first (second) sample, the tight fiducial volume cut is imposed on the cluster on the $+z(-z)$ side. The average of the two samples is used to calculate the luminosity. This procedure greatly reduces the systematic effects on the luminosity measurement due to calorimeter misalignments and $e^{+} e^{-}$ interaction point displacements. The asymmetric energy cut ensures that the acceptance is not sensitive to detector inefficiencies. In addition, most of the radiative Bhabha events are retained. Almost all the background from random beamgas coincidences has an energy less than $0.8 E_{\text {beam }}$ in each calorimeter and is therefore substantially reduced by the energy requirement.

The coplanarity requirement is used to further suppress beam related background. The remaining background in the signal region is subtracted separately for each LEP fill, using the sidebands of the coplanarity distribution, $10^{\circ}<$ $\left|\Delta \phi-180^{\circ}\right|<30^{\circ}$, after imposing the requirement that the energy on neither side is within $5 \%$ of $E_{\text {beam }}$ [3]. The residual background level of $0.1 \%$ is mainly due to random coincidences of beam-gas interactions.

Theoretical cross section. To determine the visible cross section, $e^{+} e^{-} \rightarrow e^{+} e^{-}(\gamma)$ events are simulated at a fixed centerof-mass energy, $\sqrt{s}$, of $91.18 \mathrm{GeV}$ using the event generator BHLUMI $2.01[9,10]$. For center-of-mass energies, $\sqrt{s^{\prime}}$, off the $Z$ peak, the visible cross section is rescaled by $s / s^{\prime}$. The

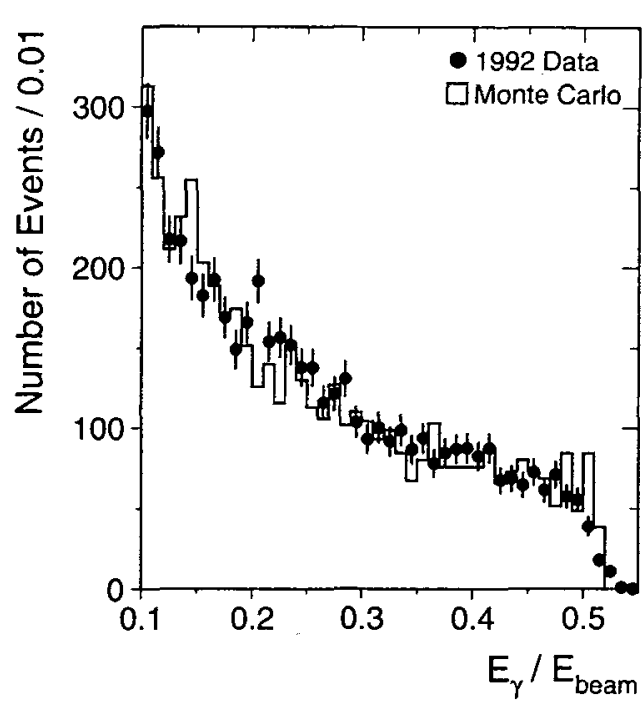

Fig. 2. The energy of photon candidates in radiative luminosity Bhabha events, $E_{\gamma}$, normalized to the beam energy, and compared to the simulated Monte-Carlo Bhabha events. Only the statistical errors on the data are shown. The statistical errors on the Monte-Carlo simulation are approximately twice as large

event generator BABAMC [11] is used to include missing electroweak corrections. A contribution of $0.02 \%$ from the process $e^{+} e^{-} \rightarrow \gamma \gamma(\gamma)$ is taken into account [12].

Including all contributions, the visible cross section at the $Z$ peak is $90.3 \mathrm{nb}$ for $1992,90.7 \mathrm{nb}$ for 1991 and $84.7 \mathrm{nb}$ for 1990 . The differences are due to shifts in the detector position along the beam axis. Inside our angular acceptance the theoretical error on the Bhabha cross section is estimated to be $0.25 \%[10,13]$. 
Table 1. Systematic uncertainties on the luminosity measurement

\begin{tabular}{ll}
\hline Source of systematic uncertainty & Contribution to $\delta \mathscr{L} / \mathscr{B}[\%]$ \\
\hline Selection cuts & 0.3 \\
Monte Carlo statistics & 0.1 \\
Geometry of the calorimeters & 0.4 \\
Background subtraction & negligible \\
Trigger inefficiency & negligible \\
\hline Total experimental systematic uncertainty & 0.5 \\
Theoretical systematic uncertainty & 0.25 \\
\hline Total systematic uncertainty & 0.6 \\
\hline
\end{tabular}

Luminosity determination. The selection quantities show good agreement between the data and the Monte-Carlo prediction (Fig. 1). The small excess of data events at high energies in the energy distribution is due to real Bhabha interactions contaminated with a spurious beam-gas interaction. Studying the effect of changes in the selection requirements on the total luminosity, we assign a systematic error of $0.3 \%$ on the luminosity due to event selection.

Radiative Bhabha events are used to investigate the quality of the Monte-Carlo event generator. The photon is identified as the smaller energy cluster in events with two separate clusters on the same side. Figure 2 shows the distribution of the measured energy, $E_{\gamma}$, of the photon candidates, and the Monte Carlo prediction. The agreement is good.

The geometry and position of the calorimeters is measured to an accuracy of $0.2 \mathrm{~mm}$ except for the position along the beam axis which is surveyed with an accuracy of 0.5 $\mathrm{mm}$. This results into a systematic uncertainty of $0.4 \%$ on the luminosity.

The luminosity trigger is described in detail in $[3,6]$. The trigger inefficiency is found to be negligible for the selected event sample.

The contributions to the systematic error on the luminosity measurement are summarized in Table 1 . Combining them in quadrature we assign a systematic error of $0.6 \%$ to the measured total luminosity.

\section{Analysis of $Z$ decays}

We analyze all the visible $Z$ decays by measuring the reactions $e^{+} e^{-} \rightarrow$ hadrons $(\gamma), e^{+} e^{-} \rightarrow \mu^{+} \mu^{-}(\gamma), e^{+} e^{-} \rightarrow$ $\tau^{+} \tau^{-}(\gamma)$, and $e^{+} e^{-} \rightarrow e^{+} e^{-}(\gamma)$. Below we describe the event selection applied to the 1991 and 1992 data and the determination of the total cross sections and forward-backward asymmetries. The selection criteria for the 1990 data differ slightly [3], as the end-cap electromagnetic calorimeter was added in 1991.

\section{$4.1 e^{+} e^{-} \rightarrow$ hadrons $(\gamma)$}

Event selection. The selection of the hadronic $Z$ decays is mainly based on calorimetric information. The process is identified by the large amount of energy deposited in the detector, and a high particle multiplicity in the final state. Energy depositions in adjacent cells of the calorimeters are grouped to form clusters [14]. Since a hadronic event contains on average about 40 clusters we easily reject the lowmultiplicity leptonic $Z$ decays. The selection criteria are:
Table 2. Systematic uncertainties on the total hadron cross section, $\sigma_{\text {had }}$ excluding the luminosity error

\begin{tabular}{ll}
\hline Source of systematic uncertainty & Contribution to $\delta \sigma_{\text {had }} / \sigma_{\text {had }}[\%]$ \\
\hline Selection cuts & 0.06 \\
Monte-Carlo acceptance and efficiency & 0.10 \\
Background subtraction & 0.08 \\
Trigger inefficiency & 0.03 \\
\hline Total systematic uncertainty & 0.15 \\
\hline
\end{tabular}

1. The total energy observed in the detector, including the momenta of muons measured in the muon spectrometer, $E_{\mathrm{vis}}$, is restricted to the range $0.5<E_{\mathrm{vis}} / \sqrt{s}<2.0$.

2 . The energy deposited in the hadron calorimeter, $E_{\text {had }}$, must be larger than $2.5 \mathrm{GeV}$.

3. The energy imbalance along the beam direction, $E_{\|}$, must satisfy $\left|E_{\|} / E_{\text {vis }}\right|<0.6$.

4. The transverse energy imbalance, $E_{\perp}$, must satisfy $E_{\perp} / E_{\mathrm{vis}}<0.5$.

5 . The number of energy clusters, $N_{\text {clusters }}$, reconstructed in the calorimeters is required to satisfy:

a) $N_{\text {ciusters }} \geq 13$ for $\left|\cos \theta_{t}\right| \leq 0.74$ (event in the barrel region),

b) $N_{\text {clusters }} \geq 17$ for $\left|\cos \theta_{t}\right|>0.74$ (event in the end-cap region),

where $\theta_{t}$ is the polar angle of the event thrust axis.

Figure 3 shows the distributions of the visible energy, the parallel and perpendicular energy imbalance, and the number of clusters, comparing the data to fully simulated MonteCarlo events. All cuts except the indicated one have been applied.

Cross section. The hadron calorimeter covers $99.5 \%$ of the full solid angle which results in a selection efficiency for $e^{+} e^{-} \rightarrow$ hadrons $(\gamma)$ events of $(99.19 \pm 0.10) \%$, determined from Monte-Carlo events. The uncertainty on this number is estimated by comparing different fragmentation models as implemented in the Monte-Carlo event generators JETSET 7.3 [15] and HERWIG 5.3 [16].

For all distributions we find a good agreement between the data and the Monte-Carlo prediction, especially in the region of the cut position. The systematic error originating from the event selection estimated by varying the above selection cuts is found to be small, $0.06 \%$. The deviation in the distribution of the number of clusters for $N_{\text {clusters }}>70$ is attributed to the imperfect simulation of low energy hadrons in the calorimeters. We have verified that the events in this region are hadronic events.

Applying the selection to $e^{+} e^{-} \rightarrow \tau^{+} \tau^{-}(\gamma)$ events simulated by the KORALZ 3.8 [17] Monte Carlo, we find a background contribution of $(0.20 \pm 0.02) \%$. The background from all other $\mathrm{Z}$ decays is negligible. The non-resonant background contribution, e.g., two-photon processes, beam-gas interactions and cosmic rays, is estimated by extrapolating the observed event rate at low visible energy, $0.2<$ $E_{\text {vis }} / \sqrt{s}<0.5$, into the signal region. We find a $\sqrt{s}$ independent contamination of $(35 \pm 15) \mathrm{pb}$. The total background subtraction leads to a systematic error on the cross section of $0.08 \%$. 

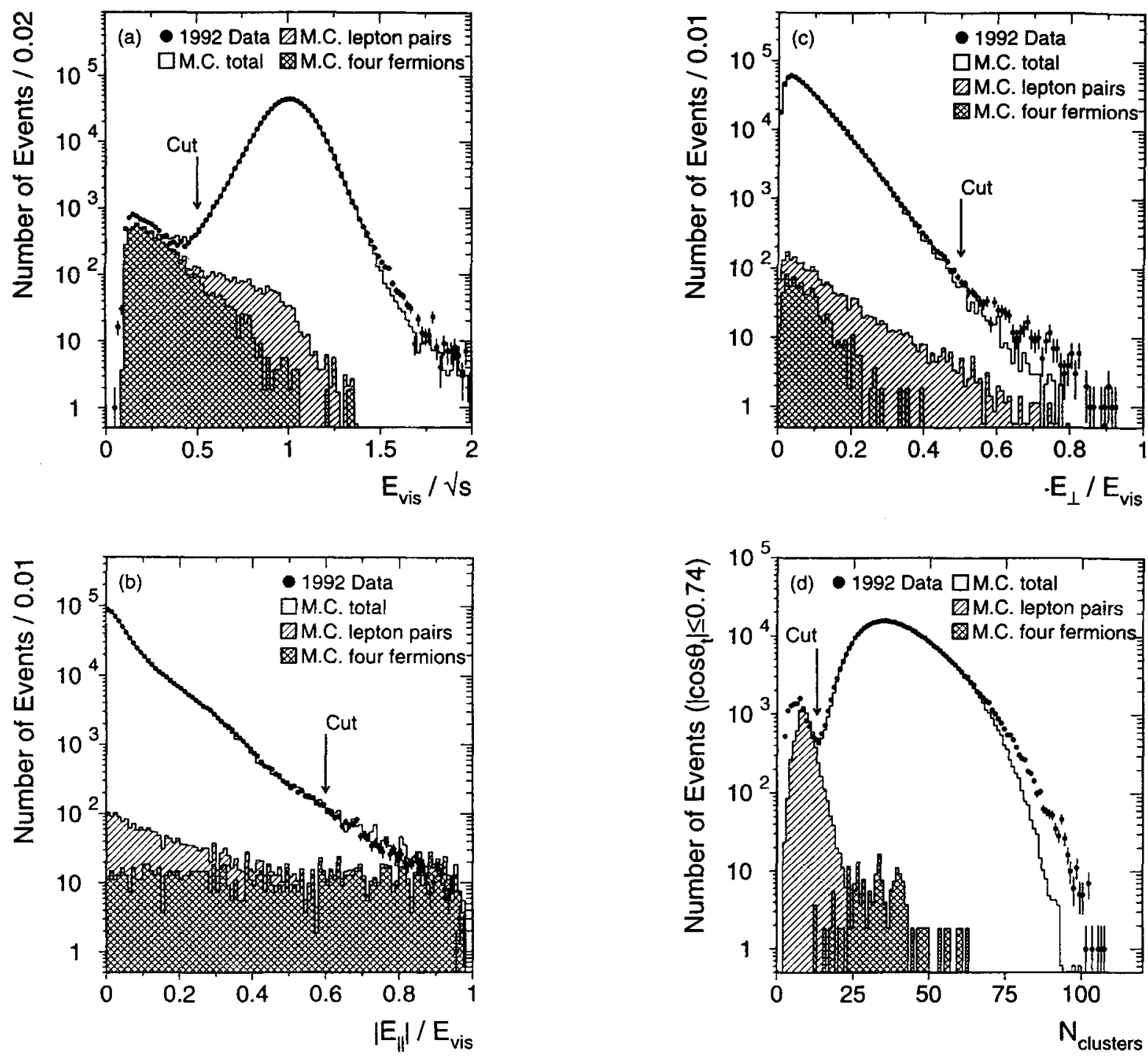

Fig. 3. a-d. Distributions of variables used for the selection of $e^{+} e^{-} \rightarrow$ hadrons $(\gamma)$ events, comparing the 1992 data to the simulated signal and background Monte-Carlo events. The position of the selection cuts are indicated by vertical arrows. a The total visible energy, $E_{\text {vis }}$, normalized to the center-ofmass energy, $\sqrt{s}$, for hadronic events. b The parallel energy imbalance, $\left|E_{\|}\right|$, normalized to the visible energy. $\mathbf{c}$ The perpendicular energy imbalance, $E_{\perp}$, normalized to the visible energy. d The number of reconstructed calorimetric clusters, $N_{\text {clusters }}$, for events with the thrust axis in the barrel region $\left(\left|\cos \theta_{t}\right| \leq 0.74\right)$

Hadronic events are triggered by the energy, central track, muon or scintillator multiplicity triggers. The cross section is corrected for the overall trigger inefficiency of $(0.16 \pm 0.03) \%$. The contributions to the systematic error on the cross-section measurement are summarized in Table 2. We assign a systematic error of $0.15 \%$ on the total cross section of the reaction $e^{+} e^{-} \rightarrow$ hadrons $(\gamma)$, excluding the error on the luminosity measurement.

The number of selected events and the total cross sections for the different center-of-mass energy points are listed in Table 9 of Sect. 5 . The cross sections are compared to the result of a fit to the complete data set in Fig. 13 of Sect. 6 .

$4.2 e^{+} e^{-} \rightarrow \mu^{+} \mu^{-}(\gamma)$
Event selection. The event selection for the process $e^{+} e^{-} \rightarrow$ $\mu^{+} \mu^{-}(\gamma)$ requires two identified muons within the fiducial angular region of $|\cos \theta|<0.8$. In the L3 detector, muons are primarily identified by a track in the muon spectrometer. For particles traversing at least 2 out of the 3 muon chamber layers, the track is reconstructed and its momentum as well as its charge is measured.

Muons are also identified by their minimum ionizing particle (MIP) signature in the inner subdetectors, if less than 2 muon chambers are hit. Including these muons in our sample makes the selection less sensitive to the exact knowledge of the uncertainties in the efficiency and geometry of individual subdetectors. Figure 4 shows a $45 \mathrm{GeV}$ muon with its typical MIP signature in the detector.

A muon candidate is denoted as a MIP, if at least one of the following conditions is fulfilled: 


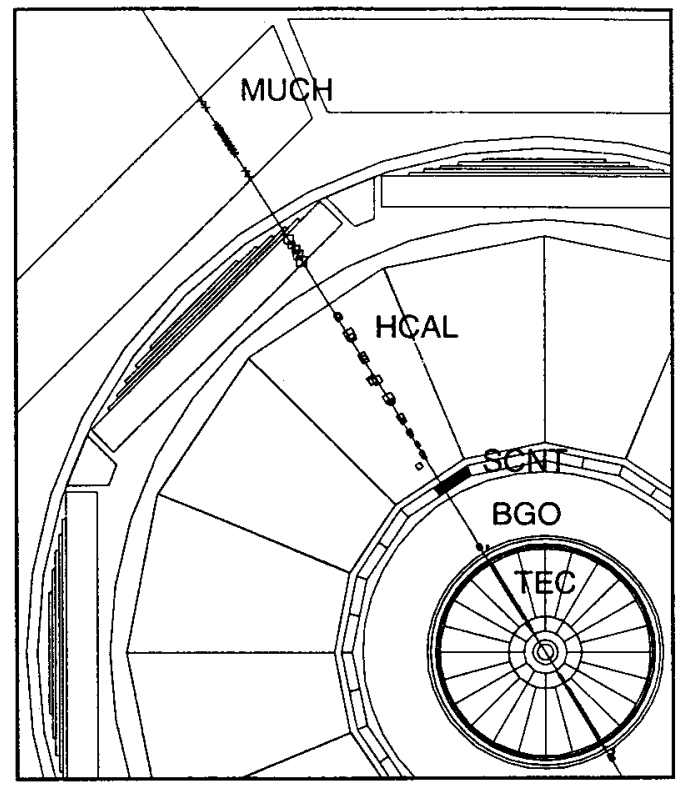

Fig. 4. A high energy muon seen in the different subdetectors of L3, with a track in the central tracking chamber (TEC), a low energy cluster in the electromagnetic calorimeter (BGO), a hit in the scintillation counters (SCNT), a track of low energy hits in the hadron calorimeter (HCAL) and hits in the inner muon chamber (MUCH)

1. A track in the central tracking chamber must point within $5^{\circ}$ in azimuth to a cluster in the electromagnetic calorimeter with an energy less than $2 \mathrm{GeV}$.

2. On a road from the vertex through the barrel hadron calorimeter, at least 5 out of a maximum of 32 cells must be hit, with an average energy of less than $0.4 \mathrm{GeV}$ per cell.

3. A track in the central chamber or a low energy electromagnetic cluster must point within $10^{\circ}$ in azimuth to a muon chamber hit.

In addition, both the electromagnetic and the hadronic energy in a cone of $12^{\circ}$ half-opening angle around the MIP candidate, corrected for the energy loss of the particle, must be less than $5 \mathrm{GeV}$.

To reject the background from hadronic $Z$ decays we require each event to have less than 15 energy clusters in the calorimeters. To reduce the cosmic-ray background at least one of the particles should have an associated scintillation counter hit in a time-of-flight corrected time window of $\pm 3 \mathrm{~ns}$ around the beam crossing. Additional cosmic-ray rejection is achieved by requiring one muon to be associated with a central chamber track, which must have a distance of closest approach to the beam axis (DCA) of less than $5 \mathrm{~mm}$. An acollinearity angle cut, $\xi<40^{\circ}$, is applied on the directions of the two muons.

To reduce the background from the $\tau^{+} \tau^{-}(\gamma)$ final states and two-photon processes, the highest momentum measured in the muon spectrometer, $P_{\max }$, must exceed $\frac{2}{3} E_{\text {beam }}$. For events without reconstructed tracks in the muon chambers, we make an acollinearity angle cut of $\xi<5^{\circ}$ and require two tracks in the central tracking chamber, one of which must have a transverse momentum larger than $3 \mathrm{GeV}$.

The final event sample consists to $81.8 \%$ of events with two tracks in the muon chambers, $11.6 \%$ with one recon-
Table 3. Systematic uncertainties on the total dimuon cross section, $\sigma_{\mu}$, excluding the luminosity error

\begin{tabular}{ll}
\hline Source of systematic uncertainty & Contribution to $\delta \sigma_{\mu} / \sigma_{\mu}[\%]$ \\
\hline Selection cuts & 0.39 \\
Monte-Carlo acceptance and efficiency & 0.25 \\
Background subtraction & 0.11 \\
Trigger inefficiency & 0.15 \\
\hline Total systematic uncertainty & 0.50 \\
\hline
\end{tabular}

structed muon chamber track, and $6.6 \%$ of events with both muons identified by their MIP signature. Figure 5 shows the distributions of the $\cos \theta$ of the two muon candidates in an event, the highest momentum reconstructed in the muon spectrometer, and the acollinearity angle, comparing the data to fully simulated Monte-Carlo events.

Cross section. The efficiency of the selection is determined from $e^{+} e^{-} \rightarrow \mu^{+} \mu^{-}(\gamma)$ Monte-Carlo events generated with KORALZ to be $(92.64 \pm 0.15) \%$ inside the fiducial volume, where the error is statistical. A systematic error of $0.10 \%$ must be added, which reflects the uncertainty in the determination of the subdetector inefficiencies.

The selection quantities show good agreement between the data and the Monte-Carlo prediction (Fig. 5). The systematic error due to the event selection is estimated by varying the selection criteria. Significant contributions arise from the cuts on the muon momentum $(0.30 \%)$ and the polar angle defining the fiducial volume $(0.25 \%)$.

Using $e^{+} e^{-} \rightarrow \tau^{+} \tau^{-}(\gamma)$ Monte-Carlo events generated by KORALZ, we determine this background to be $(1.28 \pm 0.08) \%$. The contribution of all other $Z$ decays to the background is negligible. A contribution (0.05\%) of the non-resonant $e^{+} e^{-} \rightarrow e^{+} e^{-} \mu^{+} \mu^{-}$two-photon process [18] is found only for the subsample of events with no reconstructed track in the muon chambers. The sidebands of the DCA distribution for the central chamber tracks in the region from $5 \mathrm{~mm}$ to $20 \mathrm{~mm}$ are used to determine the cosmic-ray contamination. We estimate the cosmic-ray background for the nominal value of the DCA cut, $5 \mathrm{~mm}$, to be $(0.15 \pm 0.05) \%$.

Dimuon events are triggered by the central track or muon triggers. The trigger inefficiency is negligible for the events with two tracks reconstructed in the muon spectrometer. For the samples of events with one or zero reconstructed muons in the muon chambers, we rely mainly on the charged track trigger. The trigger inefficiency is determined to be $(1.9 \pm 0.5) \%$ and $(8.0 \pm 1.5) \%$, respectively. The cross section is corrected for the overall trigger inefficiency of $(0.75 \pm 0.15) \%$.

The contributions to the systematic error on the crosssection measurement are summarized in Table 3 , including the Monte-Carlo extrapolation to the full solid angle. We assign a systematic error of $0.5 \%$ on the total cross section of the reaction $e^{+} e^{-} \rightarrow \mu^{+} \mu^{-}(\gamma)$, excluding the error on the luminosity measurement.

The number of selected events and the total cross sections for the different center-of-mass energy points are listed in Table 10 of Sect. 5. The cross sections are compared to the result of a fit to the complete data set in Fig. 14 of Sect. 6. 

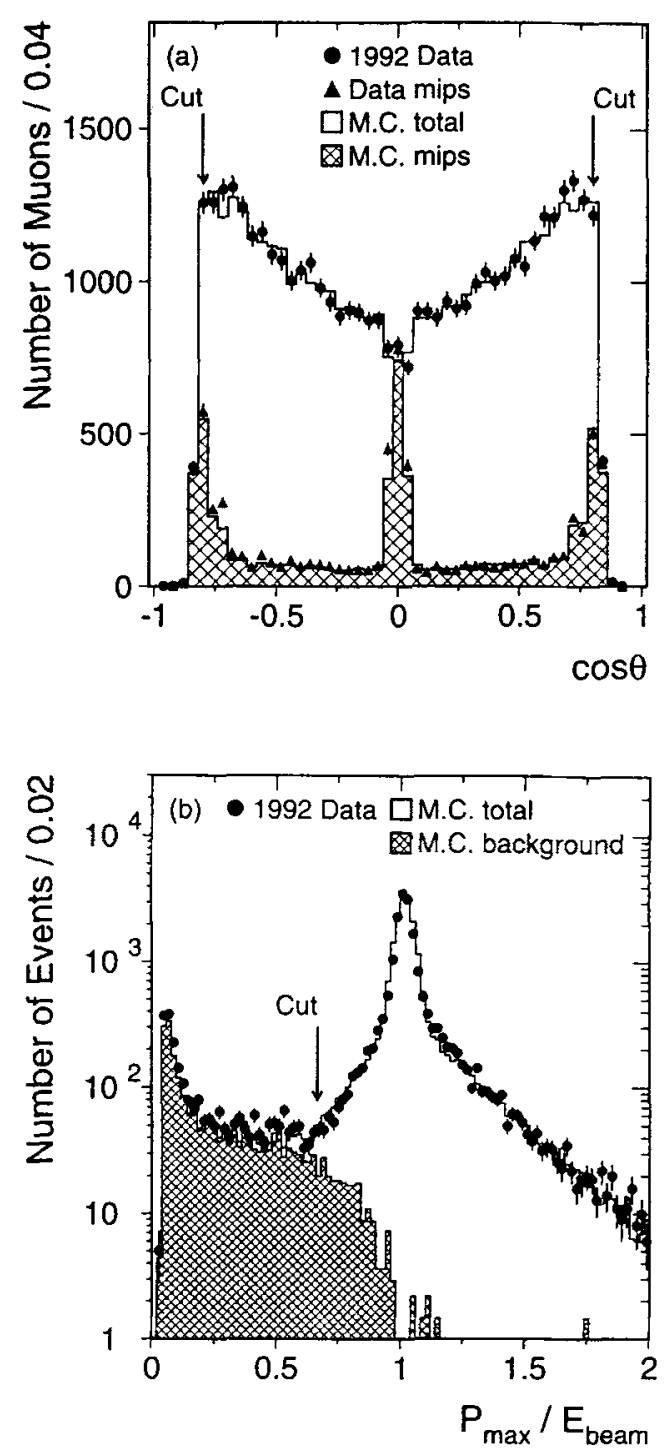
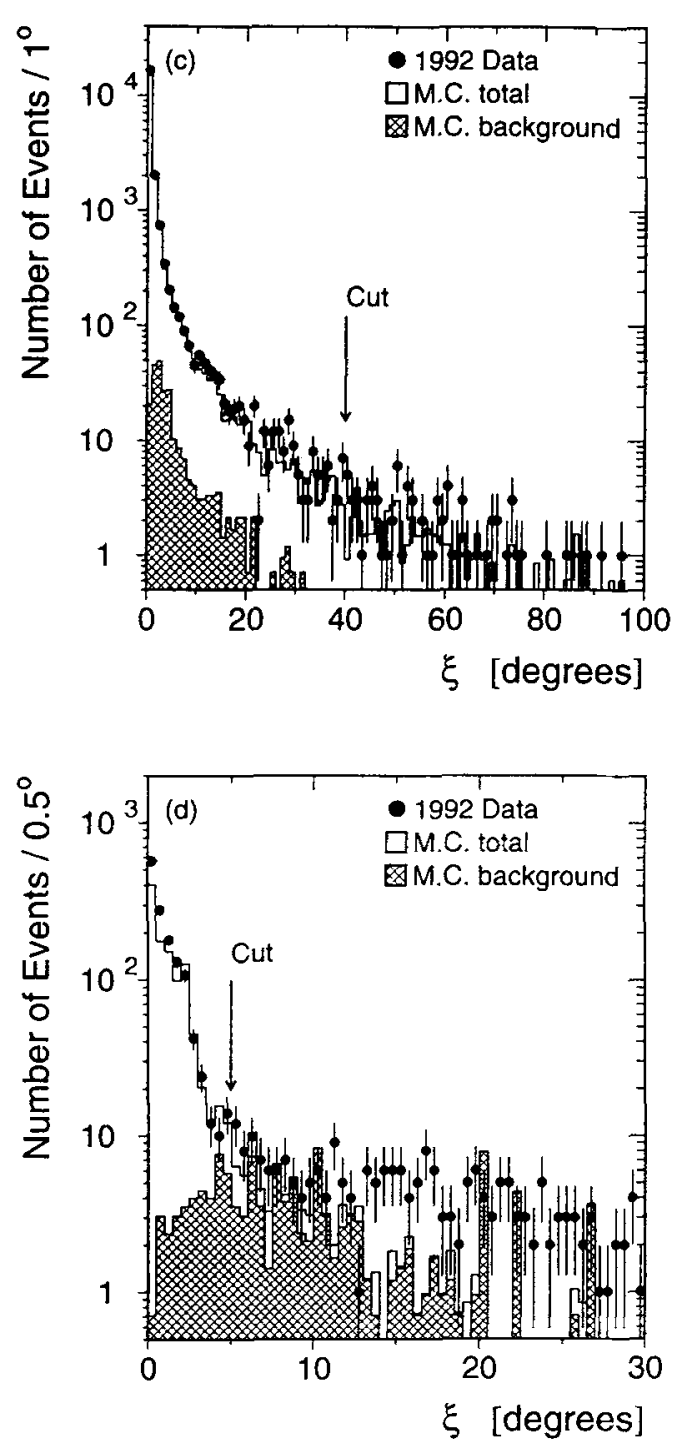

Fig. 5. a-d. Distributions of variables used for the selection of $e^{+} e^{-} \rightarrow \mu^{+} \mu^{-}(\gamma)$ events, comparing the 1992 data to the simulated signal and background Monte-Carlo events. The position of the selection cuts are indicated by vertical arrows. a The $\cos \theta$ values for both muons of an event, showing the muons identified in the muon spectrometer (87.6\%) and the muons identified as MIPs $(12.4 \%)$. b The maximum reconstructed muon momentum for events with at least one reconstructed track in the muon chambers, $P_{\max }$, normalized to the beam energy. $\mathrm{c}$ The acollinearity angle, $\xi$, between the directions of the two muons for events with at least one reconstructed track in the muon chambers. $\mathbf{d}$ Same as $\mathbf{c}$ for events with no reconstructed track in the muon chambers

Fonward-backward asymmetry. The forward-backward asymmetry, $A_{\mathrm{fb}}$, is defined as:

$A_{\mathrm{fb}}=\frac{\sigma_{\mathrm{f}}-\sigma_{\mathrm{b}}}{\sigma_{\mathrm{f}}+\sigma_{\mathrm{b}}}$

where $\sigma_{\mathrm{f}}\left(\sigma_{\mathrm{b}}\right)$ is the cross section for events with the fermion scattered into the hemisphere which is forward (backward) with respect to the $\mathrm{e}^{-}$beam direction.

Events with hard initial bremsstrahlung are removed from the sample by requiring that the acollinearity angle of the event is less than $15^{\circ}$. This allows the angular distribution in the region $|\cos \theta|<0.8$ to be approximated by the lowest-order Born formula:

$\frac{\mathrm{d} \sigma}{\mathrm{d} \cos \theta} \propto \frac{3}{8}\left(1+\cos ^{2} \theta\right)+A_{\mathrm{fb}} \cos \theta$

where $\theta$ is the polar angle of the scattered fermion with respect to the $\mathrm{e}^{-}$beam direction. A comparison with calcu- lations including higher order corrections, as implemented in the analytical program ZFITTER [19], shows that this approximation in the determination of the asymmetry does not introduce any significant systematic bias.

The asymmetry at a given center-of-mass energy point is determined by a maximum likelihood fit to our data where the likelihood function is defined as the product over the selected events labeled $i$ of the differential cross section evaluated at their respective $\cos \theta_{i}$ value:

$L \equiv \prod_{i}\left(\frac{3}{8}\left(1+\cos ^{2} \theta_{i}\right)+A_{\mathrm{fb}} \cos \theta_{i}\right)$

This method does not require an exact knowledge of the acceptance as a function of the polar angle provided that the acceptance is independent of the muon charge. 
Table 4. Systematic uncertainties on the dimuon forward-backward asymmetry, $A_{\mathrm{fb}}^{\mu}$

\begin{tabular}{ll}
\hline Source of systematic uncertainty & Contribution to $\delta A_{\mathrm{fb}}^{\mu}$ \\
\hline Charge confusion & $0.0022 A_{\mathrm{fb}}^{\mu}$ \\
Acceptance asymmetries & 0.0015 \\
Cosmic-ray background & 0.0010 \\
\hline Total systematic uncertainty & 0.0020 \\
\hline
\end{tabular}

For the measurement of the asymmetry we use only those events where at least one muon is reconstructed in the muon spectrometer. From the sample with two reconstructed muons only the events with unlike charges are used. The probability for a reversed assignment of the charges in the event is $(2.5 \pm 0.2) \cdot 10^{-5}$, derived from the number of like sign events. For the events with only one reconstructed muon the probability is $(0.85 \pm 0.14) \%$.

We have studied possible asymmetries in the acceptances for $\mu^{-}$and $\mu^{+}$in the forward or backward hemispheres. The effect of discrepancies in the observed momentum spectra on the measured asymmetry is estimated to be at most 0.0015 . Since the asymmetry of $Z$ decays into tau pairs is expected and measured to be close to that of $Z$ decays into muon pairs, the remaining background of tau-pair events does not change the observed muon asymmetry. The influence of the cosmic-ray background is small $(0.0010)$.

The contributions to the systematic error on the asymmetry measurement are summarized in Table 4 . We assign an absolute error of 0.002 on the forward-backward asymmetry of the reaction $e^{+} e^{-} \rightarrow \mu^{+} \mu^{-}(\gamma)$.

The differential cross section, $\mathrm{d} \sigma_{\mu} / \mathrm{d} \cos \theta$, of the reaction $e^{+} e^{-} \rightarrow \mu^{+} \mu^{-}(\gamma)$ for events collected at $91.29 \mathrm{GeV}$ (1992 data) is shown in Fig. 6. The result for the forwardbackward asymmetry obtained from a fit to this distribution agrees with the result from the maximum-likelihood method. The forward-backward asymmetries for the different centerof-mass energy points are listed in Table 13 of Sect. 5. The asymmetries are compared to the result of a fit to the complete data set in Fig. 17 of Sect. 6.

$4.3 e^{+} e^{-} \rightarrow \tau^{+} \tau^{-}(\gamma)$

Event selection. The visible decay products of a tau form a jet, which consists of either an electron, a muon, a charged hadron or a few highly collimated charged and neutral hadrons. Although there does not exist a unique event signature, the aim is to select $\tau^{+} \tau^{-}(\gamma)$ events of all tau decay modes. This is achieved by excluding other final states from a sample of selected $Z$ decays.

Tau pairs are selected within a fiducial volume defined by $\left|\cos \theta_{t}\right|<0.73$ where the polar angle, $\theta_{t}$, is given by the thrust axis of the event. The event is required to have at least two jets, corresponding to the two taus, with minimal visible energies of 7 and $3 \mathrm{GeV}$. The acollinearity angle between the directions of the two highest energy jets, $\xi$, must be smaller than $14.3^{\circ}$.

High multiplicity hadronic $Z$ decays are removed by requiring less than 13 reconstructed energy clusters in the calorimeters. In addition, there should be no track in the central tracking chamber with an azimuthal angle, $\Delta \phi$, larger

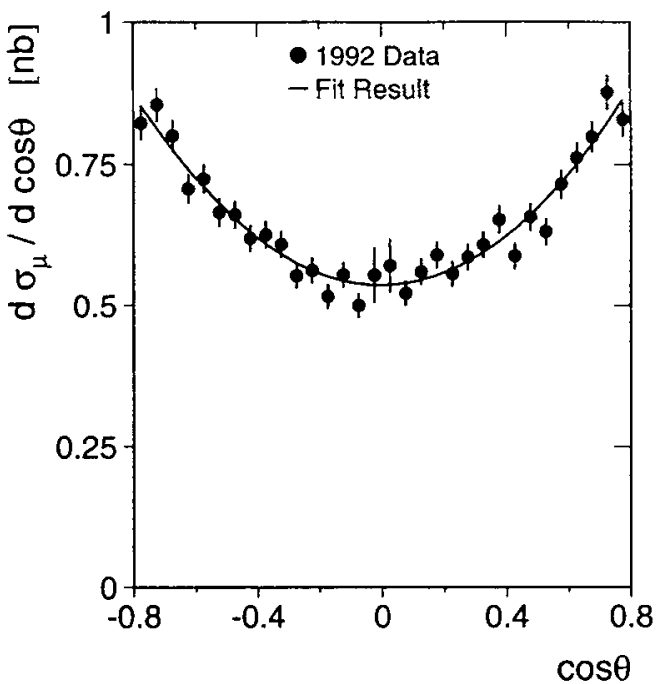

Fig. 6. The differential cross section, $\mathrm{d} \sigma_{\mu} / \mathrm{d} \cos \theta$, as a function of the polar scattering angle, $\cos \theta$, of the final-state fermion for $e^{+} e^{-} \rightarrow \mu^{+} \mu^{-}(\gamma)$ events on the $Z$ peak (1992 data)

than $14.3^{\circ}$ with respect to the axis of the nearest jet. In order to reject $e^{+} e^{-} \rightarrow e^{+} e^{-}(\gamma)$ events, the two highest energy clusters in the electromagnetic calorimeter with an electromagnetic shower shape must have energies below $0.90 E_{\text {beam }}$ and $0.65 E_{\text {beam }}$. In order to reduce the background of $e^{+} e^{-} \rightarrow \mu^{+} \mu^{-}(\gamma)$ events, the momentum measured in the muon chambers must be less than $0.9 E_{\text {beam }}$ for the highest energy, and $0.4 E_{\text {beam }}$ for the second highest energy muon candidate in the event. The requirement of at least $2 \mathrm{GeV}$ of energy deposited in the electromagnetic calorimeter rejects dimuon events, where the muons are not reconstructed in the muon chambers, as well as minimum ionizing cosmicray events. The cosmic-ray background is further reduced by requiring a scintillation counter hit within $2.5 \mathrm{~ns}$ of the beam crossing.

Figure 7 shows the distributions of the acollinearity angle between the two jet directions and the largest azimuthal angle between a track in the central chamber and the axis of its nearest jet, comparing the data to fully simulated MonteCarlo events.

Cross section. The efficiency of the selection is determined from $e^{+} e^{-} \rightarrow \tau^{+} \tau^{-}(\gamma)$ Monte-Carlo events generated with KORALZ to be $(78.79 \pm 0.11) \%$ inside the fiducial volume, where the error is statistical. Because the acceptance depends on the decay modes of the two taus in the event, an additional systematic uncertainty of $0.25 \%$ must be added due to the uncertainties on the tau branching fractions.

The distribution of the number of clusters is evaluated from the data based on a sample of tau-pair events selected using the multiplicity of tracks in the central chamber instead of clusters in the calorimeters. This study leads to a correction on the total cross section of $(0.8+0.4) \%$. The other selection quantities show good agreement between the data and the Monte-Carlo prediction (Fig. 7). Their systematic uncertainties are derived from varying the corresponding 

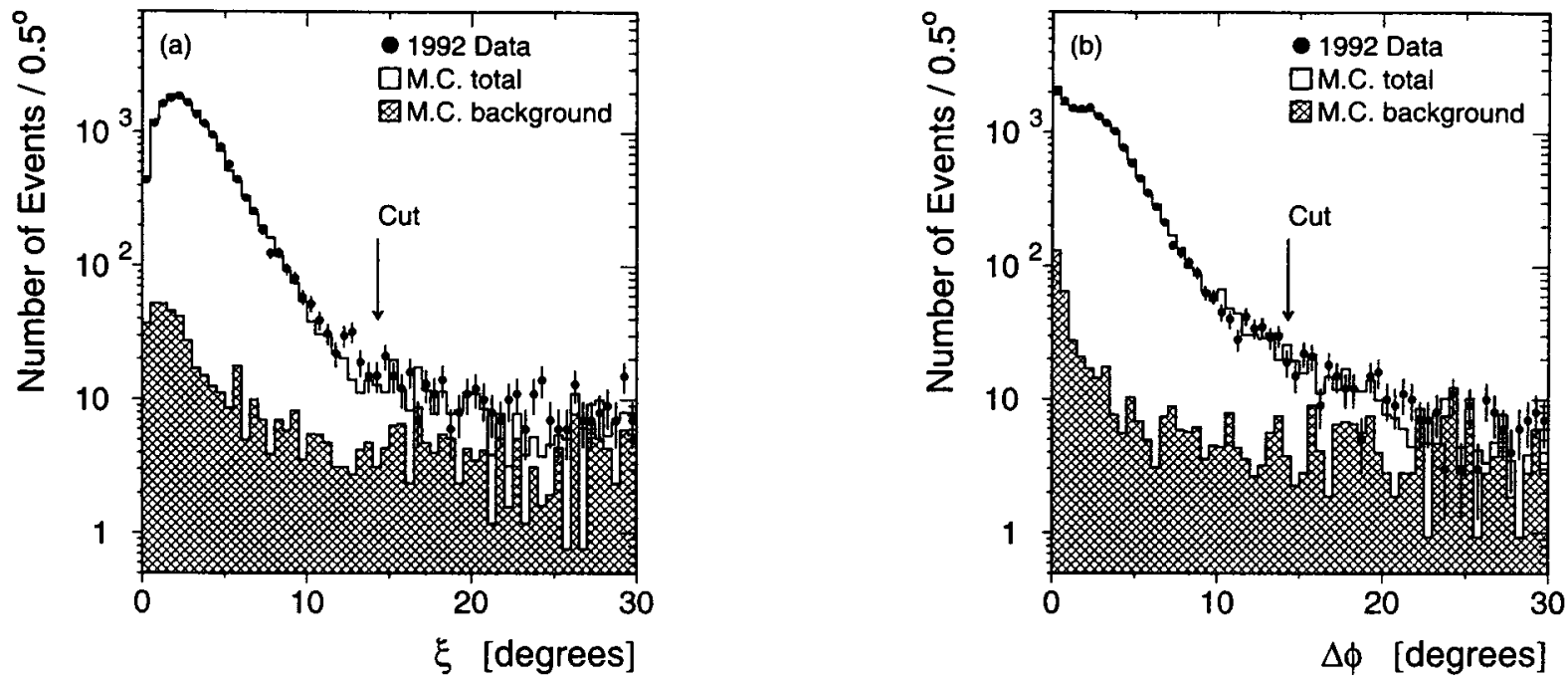

Fig. 7. Distributions of variables used for the selection of $e^{+} e^{-} \rightarrow \tau^{+} \tau^{-}(\gamma)$ events, comparing the 1992 data to the simulated signal and background Monte-Carlo events. The position of the selection cuts are indicated by vertical arrows. a The acollinearity angle, $\xi$, between the directions of the two highest energy jets. b The maximal separation in azimuth, $\Delta \phi$, between a track in the central tracking chamber and the nearest jet

Table 5. Systematic uncertainties on the total tau-pair cross section, $\sigma_{\tau}$, excluding the luminosity error

\begin{tabular}{ll}
\hline Source of systematic uncertainty & Contribution to $\delta \sigma_{\tau} / \sigma_{\tau}[\%]$ \\
\hline Selection cuts & 0.60 \\
Monte-Carlo acceptance and efficiency & 0.21 \\
Tau-decay branching fractions & 0.25 \\
Background subtraction & 0.14 \\
Trigger inefficiency & 0.05 \\
\hline Total systematic uncertainty & 0.70
\end{tabular}

cuts. A total systematic error of $0.6 \%$ is assigned to the event selection.

In this sample a background of $(2.75 \pm 0.12) \%$, determined by Monte Carlo, remains from the other $Z$ decay channels. The contamination of cosmic rays is estimated to be $(0.15 \pm 0.05) \%$. The background from two-photon processes is negligible, $(0.9 \pm 0.5) \mathrm{pb}$.

Tau-pair events are triggered by the energy, central track, muon or scintillator multiplicity triggers. The cross section is corrected for the overall trigger inefficiency of $(0.10 \pm 0.05) \%$. The contributions to the systematic error on the cross-section measurement are summarized in Table 5, including the Monte-Carlo extrapolation to the full solid angle. We assign a systematic error of $0.7 \%$ on the total cross section of the reaction $e^{+} e^{-} \rightarrow \tau^{+} \tau^{-}(\gamma)$, excluding the error on the luminosity measurement.

The number of selected events and the total cross sections for the different center-of-mass energy points are listed in Table 11 of Sect. 5. The cross sections are compared to the result of a fit to the complete data set in Fig. 15 of Sect. 6 .

Forward-backward asymmetry. The determination of the forward-backward asymmetry is carried out in the same way as for the $e^{+} e^{-} \rightarrow \mu^{+} \mu^{-}(\gamma)$ events, i.e., independent of the acceptance at each value of the scattering angle. The charge

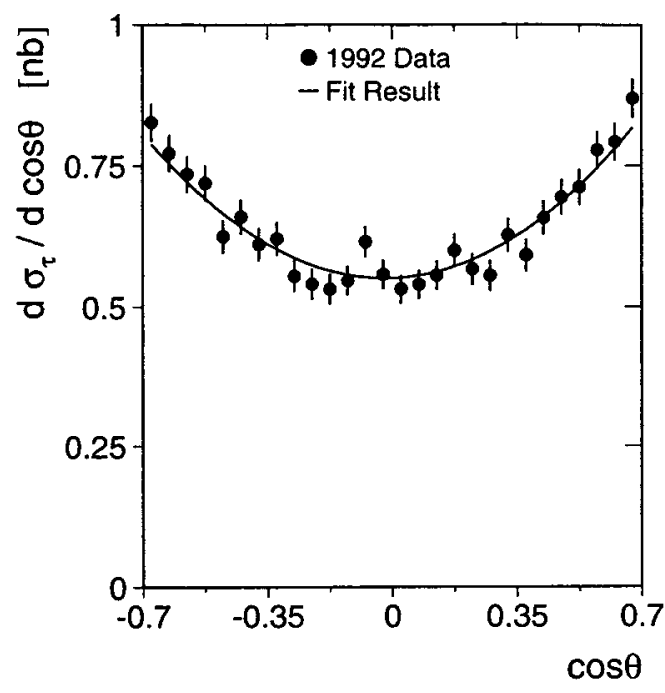

Fig. 8. The differential cross section, $\mathrm{d} \sigma_{\tau} / \mathrm{d} \cos \theta$, as a function of the polar scattering angle, $\cos \theta$, of the final-state fermion for $e^{+} e^{-} \rightarrow \tau^{+} \tau^{-}(\gamma)$ events on the $Z$ peak (1992 data)

of a tau is derived from the sum of the charges of its decay products as measured in the central tracking chamber. As the background of $e^{+} e^{-} \rightarrow e^{+} e^{-}(\gamma)$ events is mainly concentrated at the edges of the barrel electromagnetic calorimeter, events used in the asymmetry determination are restricted to $\left|\cos \theta_{t}\right|<0.7$.

For the determination of the forward-backward asymmetry we take only those events where the charges of the two tau jets have unequal sign ( $85 \%$ of the 1992 data sample). The probability for a reversed assignment of the charges in these events is determined from the ratio of like and unlike sign events in the data to be $(1.20 \pm 0.05) \%$. We correct the observed asymmetry for the charge confusion and assign a 
Table 6. Systematic uncertainties on the tau-pair forward-backward asymmetry, $A_{\mathrm{fb}}^{\tau}$

\begin{tabular}{ll}
\hline Source of systematic uncertainty & Contribution to $\delta A_{\mathrm{fb}}^{\tau}$ \\
\hline Charge confusion & $0.001 A_{\mathrm{fb}}^{\tau}$ \\
Fiducial volume & 0.003 \\
Cosmic-ray background & 0.001 \\
\hline Total systematic uncertainty & 0.003 \\
\hline
\end{tabular}

residual systematic error on the asymmetry of $0.001 A_{\mathrm{fb}}^{\tau}$ due to charge confusion.

The systematic uncertainty due to the subtraction of the $e^{+} e^{-} \rightarrow e^{+} e^{-}(\gamma)$ background in conjunction with a variation of the fiducial volume cut in $\left|\cos \theta_{t}\right|$ is estimated to be at most 0.003 . Since the asymmetry of $Z$ decays into muon pairs is expected and measured to be close to that of $Z$ decays into tau pairs, the remaining background of dimuon events does not change the observed tau asymmetry. The uncertainty introduced by the cosmic-ray background is less than 0.001 .

The contributions to the systematic error on the asymmetry measurement are summarized in Table 6 . We assign an absolute error of 0.003 on the forward-backward asymmetry of the reaction $e^{+} e^{-} \rightarrow \tau^{+} \tau^{-}(\gamma)$.

The differential cross section, $\mathrm{d} \sigma_{\tau} / \mathrm{d} \cos \theta$, of the reaction $e^{+} e^{-} \rightarrow \tau^{+} \tau^{-}(\gamma)$ for events collected at $91.29 \mathrm{GeV}$ (1992 data) is shown in Fig. 8. The result for the forwardbackward asymmetry obtained from a fit to this distribution agrees with the result from the maximum-likelihood method. The forward-backward asymmetries for the different centerof-mass energy points are listed in Table 13 of Sect. 5. The asymmetries are compared to the result of a fit to the complete data set in Fig. 18 of Sect. 6.

$4.4 e^{+} e^{-} \rightarrow e^{+} e^{-}(\gamma)$

Event selection. The selection of $e^{+} e^{-} \rightarrow e^{+} e^{-}(\gamma)$ events makes use of the fact that such events deposit a large amount of electromagnetic energy concentrated in a small number of BGO clusters. The events must satisfy at least one of the following three requirements:

1. There must be at least two clusters in the electromagnetic calorimeter. The highest energy cluster must have an energy measured in the $\mathrm{BGO}, E_{\max }$, larger than $0.9 E_{\text {beam }}$. The second cluster must have an energy larger than $2 \mathrm{GeV}$.

2. The sum of the energy of the four highest energy clusters in the electromagnetic calorimeter, $E_{\mathrm{BGO}}$, must be larger than $70 \%$ of the center-of-mass energy.

3 . When there is no second cluster in the electromagnetic calorimeter with an energy larger than $2 \mathrm{GeV}$, we require a cluster in the hadron calorimeter with electromagnetic shower shape and at least $7.5 \mathrm{GeV}$ energy opposite to the leading cluster. This recovers events $(0.4 \%$ of the final sample) with energy leaking through the BGO support structure.

The acollinearity angle, $\xi$, between the directions of the two clusters of highest energy is required to be less than $25^{\circ}$. We also require less than 15 clusters in total to suppress the hadronic $Z$ decays.
Table 7. Systematic uncertainties on the $e^{+} e^{-} \rightarrow e^{+} e^{-}(\gamma)$ cross section, $\sigma_{\mathfrak{e}}$, excluding the luminosity error

\begin{tabular}{ll}
\hline Source of systematic uncertainty & Contribution to $\delta \sigma_{\mathrm{e}} / \sigma_{\mathrm{e}}[\%]$ \\
\hline Selection cuts & 0.26 \\
Monte-Carlo acceptance and efficiency & 0.11 \\
Background subtraction & 0.13 \\
Trigger inefficiency & 0.01 \\
\hline Total systematic uncertainty & 0.31 \\
\hline
\end{tabular}

The cross section and forward-backward asymmetry are measured at large polar angles, where the relative contribution of the s-channel $\mathrm{Z}$ exchange is dominant.

Two event samples are maintained as in the luminosity analysis. The first sample consists of the selected events where the highest energy cluster on the $+z$ side has a polar angle $\theta>44.69^{\circ}$. The second sample consists of the selected events where the highest energy cluster on the $-z$ side has a polar angle $\theta<135.31^{\circ}$. These angles correspond to a distance of 1.5 crystal widths away from the edges of the barrel electromagnetic calorimeter. The average cross section of the two samples has a reduced sensitivity to beam offsets and differences in the beam spot shapes between data and Monte-Carlo events. A symmetric fiducial volume cut, $44^{\circ}<\theta<136^{\circ}$, for the two highest energy clusters, which excludes the outermost ring of barrel crystals, gives consistent results once the beam spot parameters in the simulation are tuned to the experimental values.

Figure 9 shows the distribution of the energy of the highest energy cluster and the energy sum of the four highest energy clusters, comparing the data to fully simulated MonteCarlo events.

Cross section. The efficiency of the selection, $(97.29 \pm 0.10) \%$, is determined using Monte-Carlo events generated with the program BHAGENE3 [20], which generates up to three radiative photons in the final state. As a cross-check we also use events generated to first order with the program BABAMC [11]. The efficiencies estimated with the two event generators agree to within $0.1 \%$.

The exact knowledge of the geometry of the barrel electromagnetic calorimeter is important for the angular cuts. By comparing the survey measurements with the data from the central tracking chamber and the hadron calorimeter we estimate the error in the definition of the fiducial volume to be less than $0.05^{\circ}$, which corresponds to a systematic error of $0.15 \%$ in the measured cross section.

The selection quantities show good agreement between the data and the Monte-Carlo prediction (Fig. 9). The systematic uncertainties of the selection are estimated from variations of the cuts around their nominal values. The energy cuts contribute with $0.21 \%$ and the angular cuts with $0.16 \%$ to the total systematic error.

The background, estimated using fully simulated Monte Carlo events, consists of $(1.53 \pm 0.09) \% \tau^{+} \tau^{-}(\gamma)$ events and $(16.4 \pm 0.1) \mathrm{pb}$ of $e^{+} e^{-} \rightarrow \gamma \gamma(\gamma)$ events at the $\mathrm{Z}$ peak. The background of hadronic events is below $0.1 \%$.

The $e^{+} e^{-} \rightarrow e^{+} e^{-}(\gamma)$ events are triggered by the energy, central track or scintillator multiplicity triggers. The cross section is corrected for the overall trigger inefficiency of 

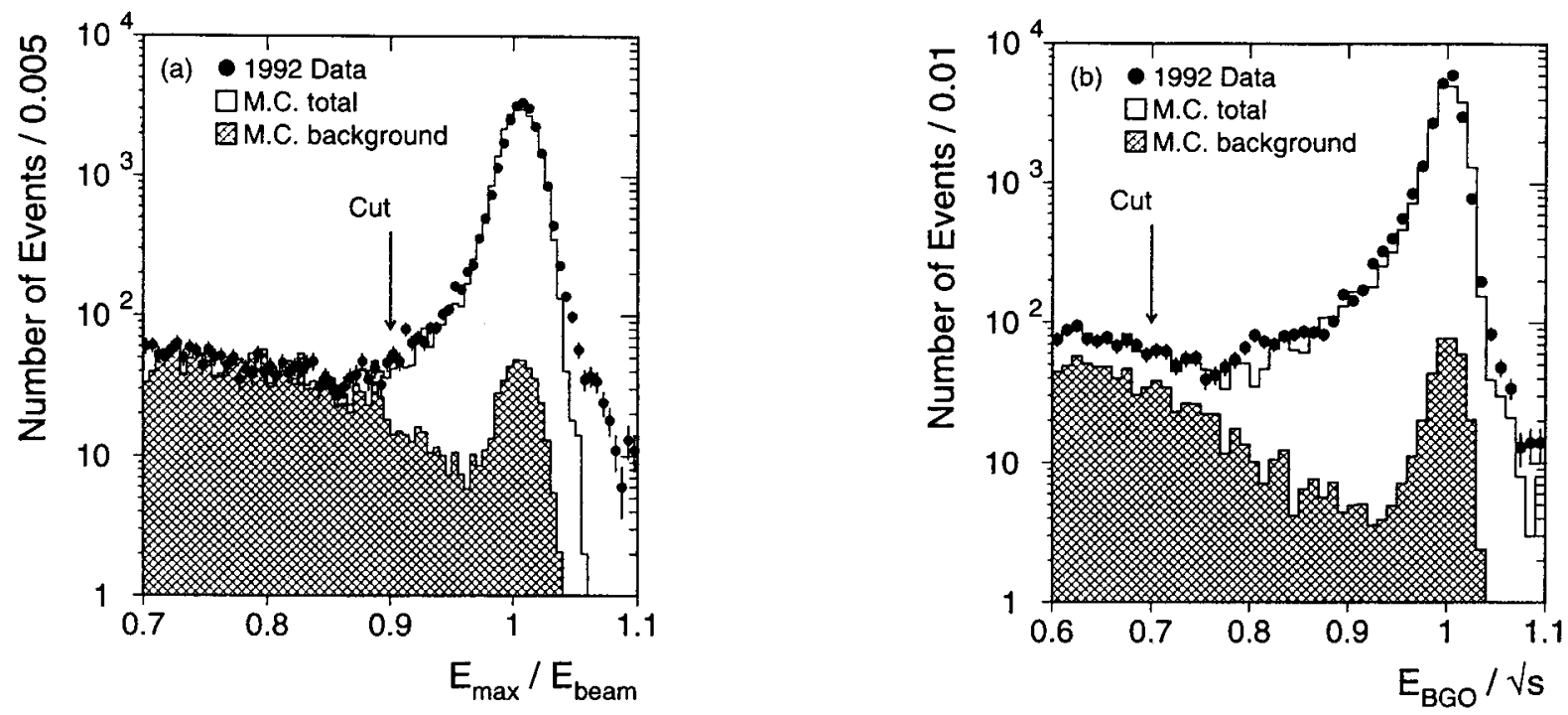

Fig. 9. a,b Distributions of variables used for the selection of $e^{+} e^{-} \rightarrow e^{+} e^{-}(\gamma)$ events, comparing the 1992 data to the simulated signal and background Monte-Carlo events. The position of the selection cuts are indicated by vertical arrows. a The energy of the highest energy cluster in the electromagnetic calorimeter, $E_{\max }$, normalized to the beam energy. $\mathbf{b}$ The energy sum of the four highest energy clusters in the electromagnetic calorimeter, $E_{\mathrm{BGO}}$, normalized to the center-of-mass energy. The background at (a) $E_{\max } \approx E_{\text {beam }}$ and (b) $E_{\mathrm{BGO}} \approx \sqrt{s}$ is due to the process $e^{+} e^{-} \rightarrow \gamma \gamma(\gamma)$
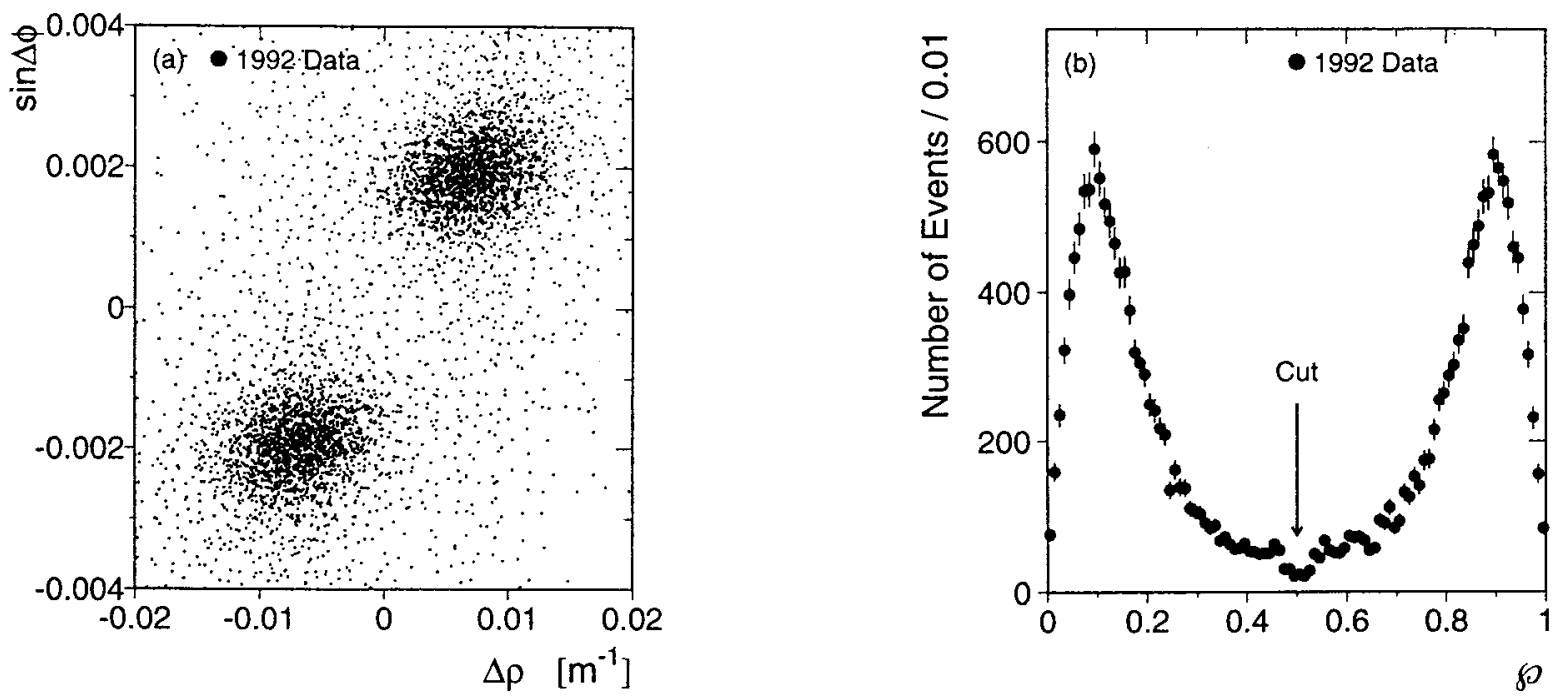

Fig. 10. a,b Distributions of quantities used for the charge assignment in $e^{+} e^{-} \rightarrow e^{+} e^{-}(\gamma)$ events. a Scatter plot of $\sin \Delta \phi$ versus $\Delta \rho$ (defined in the text) for all events selected for the forward-backward asymmetry measurement. The upper right cluster, $\mathrm{C}_{+}$, corresponds to events with the higher energy cluster being a positron, the lower left cluster, $\mathrm{C}_{-}$, to events where it is an electron. $\mathbf{b}$ Distribution of the quantity $\mathscr{P}=d_{-} /\left(d_{+}+d_{-}\right)$calculated from the weighted distances, $d_{ \pm}$, of the event to the centers of the two clusters, $\mathrm{C}_{ \pm}$, as explained in the text

$(0.06 \pm 0.01) \%$. The contributions to the systematic error on the cross-section measurement are summarized in Table 7. We assign a systematic error of $0.3 \%$ on the total cross section of the reaction $e^{+} e^{-} \rightarrow e^{+} e^{-}(\gamma)$, excluding the error on the luminosity measurement.

The number of selected events and the cross sections for the different center-of-mass energy points are listed in Table 12 of Sect. 5 . The measured cross sections are compared to the result of a fit to the complete data set in Fig. 16 of Sect. 6.
Forward-backward asymmetry. The asymmetry measurement uses the events passing the symmetric fiducial volume cut. The charges of the outgoing particles are determined by the tracks in the central tracking chamber. We require two tracks corresponding to the two leptons. In the plane transverse to the beam direction, one of the two has to match to the highest energy cluster within $25 \mathrm{mrad}$ and the second track to the second cluster within $50 \mathrm{mrad}$. We define two separators which should have positive values if the first track is a positron and negative values if it is an electron:

1. $\Delta \rho=\rho_{1}-\rho_{2}$, where $\rho=\hat{\rho} \sin \theta$. This separator is the difference of the signed curvatures, $\hat{\rho} \propto q / p_{\perp}$, corrected for their $\theta$ dependence, of the two tracks as measured by 


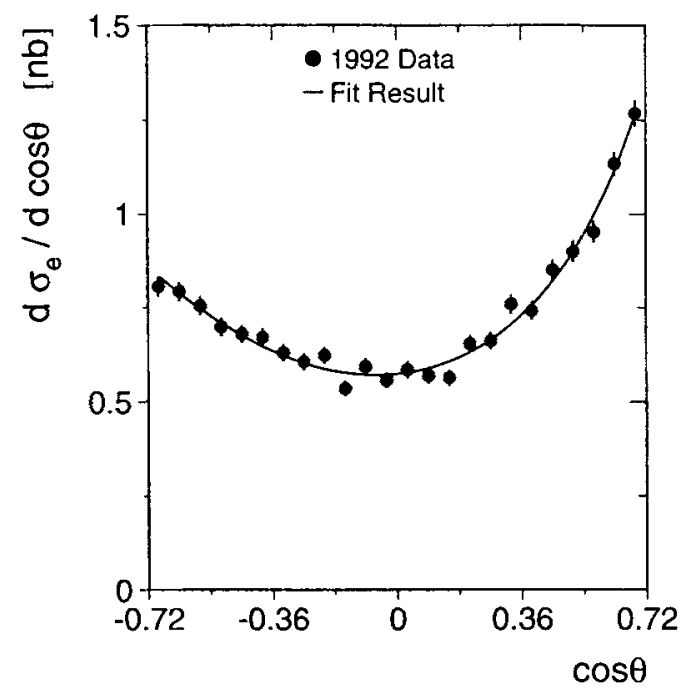

Fig. 11. The differential cross section, $\mathrm{d} \sigma_{e} / \mathrm{d} \cos \theta$, as a function of the polar scattering angle, $\cos \theta$, of the final-state fermion for $e^{+} e^{-} \rightarrow e^{+} e^{-}(\gamma)$ events on the $Z$ peak (1992 data)

the central tracking chamber. For single tracks of $45 \mathrm{GeV}$ momentum, the lever arm of $31 \mathrm{~cm}$ in the central chamber leads to a resolution on the curvature of $75 \%$ after including the average interaction point as a constraint in the track fit.

2. $\sin \Delta \phi=\sin \left(\hat{\phi}_{1}-\hat{\phi}_{2}\right)\left(\sin \theta_{1}+\sin \theta_{2}\right) / 2$, where $\hat{\phi}$ is the azimuthal angle of a straight line fitted to the hits forming a track. The factor $\left(\sin \theta_{1}+\sin \theta_{2}\right) / 2$ corrects for the $\theta$ dependence of the difference in these azimuthal angles. This separator, exploiting the good angular resolution of the central tracking chamber, is useful for events without hard photon radiation.

As shown in Fig. 10a, the events cluster in the $(\Delta \rho, \sin \Delta \phi)$ plane around two centers, $\mathrm{C}_{+}$and $\mathrm{C}_{-}$, with coordinates $\mathrm{C}_{ \pm}=$ $\pm\left(7.1 \cdot 10^{-3} \mathrm{~m}^{-1}, 1.9 \cdot 10^{-3}\right)$. The Gaussian widths of the clusters in the coordinates, $\Delta \rho$ and $\sin \Delta \phi$, are $\sigma_{\Delta \rho}=3.4$. $10^{-3} \mathrm{~m}^{-1}$ and $\sigma_{\Delta \phi}=0.56 \cdot 10^{-3}$, respectively. These widths are used to calculate the distances, $d_{+}$and $d_{-}$, of each event in the $\left(\Delta \rho / \sigma_{\Delta \rho}, \sin \Delta \phi / \sigma_{\Delta \phi}\right)$ plane to the two centers. The quantity $\mathscr{P}=d_{-} /\left(d_{+}+d_{-}\right)$, whose distribution is shown in Fig. 10b, determines the charge flow in the event. If $\mathscr{P}>$ 0.5 , meaning the event is closer to $\mathrm{C}_{+}$than to $\mathrm{C}_{-}$, the first track is recognized as a positron. If $\mathscr{P}<0.5$, meaning the event is closer to $\mathrm{C}_{-}$, the first track is recognized as an electron.

This procedure has been studied with the $\mu^{+} \mu^{-}(\gamma)$ sample where the charge is determined independently with much higher precision by the muon spectrometer. We have reversed charge assignment for $(3.1 \pm 0.2) \%$ of the events in the 1992 data sample and for $(4.0 \pm 0.3) \%$ of the events in the 1991 data sample. As this charge confusion is the main source of systematic error in the asymmetry measurement we have studied a subset of the events with much lower charge confusion by selecting events with tracks outside the lower precision regions close to the cathode and anode planes of the central tracking chamber. After correction for the reversed charge assignment the asymmetries of the two samples agree to within 0.0020 .
Table 8. Systematic uncertainties on the $e^{+} e^{-} \rightarrow e^{+} e^{-}(\gamma)$ forwardbackward asymmetry, $A_{\mathrm{fb}}^{e}$

\begin{tabular}{ll}
\hline Source of systematic uncertainty & Contribution to $\delta A_{\mathrm{fh}}^{\mathrm{e}}$ \\
\hline Charge confusion & 0.0020 \\
Width of interaction region in $z$ & 0.0010 \\
Tau-pair background & 0.0005 \\
\hline Total systematic uncertainty & 0.0023 \\
\hline
\end{tabular}

The polar angles of the scattered leptons are determined from the reconstructed center of the cluster in the electromagnetic calorimeter and the average position of the $e^{+} e^{-}$ interaction point. The polar angle, $\theta$, is measured with a resolution of $1^{\circ}$, which is dominated by the longitudinal width of the interaction region, $\sigma_{z}=8 \mathrm{~mm}$.

The forward-backward asymmetry, $A_{\mathrm{fb}}^{\mathrm{e}}$, is measured by counting the events in the forward and backward hemispheres. The data are corrected bin-by-bin for the $\cos \theta$ dependent acceptance and charge confusion. The efficiency of the track selection is estimated using the data itself by comparing the events selected for the asymmetry measurement and for the cross-section measurement, where only calorimetric information is used. To reduce the sensitivity to beam spot offsets, the asymmetry is measured twice using the scattered $\mathrm{e}^{-}$or the $\mathrm{e}^{+}$. The average of the two measurements determines the asymmetry, $A_{\mathrm{fb}}^{\mathrm{e}}$, of the event sample.

The contributions to the systematic error on the asymmetry measurement are summarized in Table 8 . We assign an absolute error of 0.002 on the forward-backward asymmetry of the reaction $e^{+} e^{-} \rightarrow e^{+} e^{-}(\gamma)$.

The differential cross section, $\mathrm{d} \sigma_{e} / \mathrm{d} \cos \theta$, of the reaction $e^{+} e^{-} \rightarrow e^{+} e^{-}(\gamma)$ for events collected at $91.29 \mathrm{GeV}(1992$ data) is shown in Fig. 11. The forward-backward asymmetries for the different center-of-mass energy points are listed in Table 13 of Sect. 5. The measured asymmetries are compared to the result of a fit to the complete data set in Fig. 19 of Sect. 6.

\section{Cross-section and forward-backward asymmetry results}

Tables 9 to 13 summarize our measurements of the cross sections and forward-backward asymmetries from the 1990, 1991 and 1992 data. These are used as input to the analysis described in Sect. 6. The quoted cross sections are total cross sections extrapolated to the full solid angle of $4 \pi$, except for the process $e^{+} e^{-} \rightarrow e^{+} e^{-}(\gamma)$, where the measured total cross sections, $\sigma_{\mathrm{e}}$, and the measured forward-backward asymmetries, $A_{\mathrm{fb}}^{\mathrm{e}}$, are within a restricted fiducial volume of $44^{\circ}<\theta<136^{\circ}$ and with an acollinearity-angle cut of $\xi<25^{\circ}$.

For completeness, we also give the s-channel contribution to the process $e^{+} e^{-} \rightarrow e^{+} e^{-}(\gamma)$ extrapolated to the full solid angle. Using the analytical program ALIBABA [21], which calculates this process in the framework of the SM, we correct the measured total cross sections and forwardbackward asymmetries for the $t$-channel and $s / t$-interference contributions expected in the SM. The calculations to derive the extrapolated s-channel cross section, $\sigma_{\mathrm{e}}^{\mathrm{s}}$, and its statistical error, $\delta \sigma_{\mathrm{e}}^{\mathrm{s}}$, are as follows: 
Table 9. Cross sections for $e^{+} e^{-} \rightarrow$ hadrons $(\gamma)$, extrapolated to the full solid angle. The quoted systematic error excludes the uncertainty of $0.6 \%$ in the luminosity

\begin{tabular}{|c|r|r|r|}
\hline \multicolumn{5}{|c|}{1990 Data } \\
\hline$\sqrt{s}[\mathrm{GeV}]$ & $N_{\text {events }}$ & $\mathscr{L}\left[\mathrm{nb}^{-1}\right]$ & $\sigma_{\text {had }}[\mathrm{nb}]$ \\
\hline 88.231 & 1525 & 339.4 & $4.46 \pm 0.12$ \\
89.236 & 3600 & 422.9 & $8.52 \pm 0.15$ \\
90.238 & 6147 & 330.9 & $18.68 \pm 0.26$ \\
91.230 & 79157 & 2624.0 & $30.44 \pm 0.13$ \\
92.226 & 8182 & 374.4 & $22.01 \pm 0.27$ \\
93.228 & 5922 & 480.3 & $12.38 \pm 0.17$ \\
94.223 & 3752 & 465.7 & $8.06 \pm 0.14$ \\
\hline Totals & 108285 & 5037.6 & \\
\hline Systematic uncertainty & & $\pm 0.3 \%$ \\
\hline
\end{tabular}

\begin{tabular}{|c|r|r|r|}
\hline \multicolumn{5}{|c|}{ 1991 Data } \\
\hline$\sqrt{s}[\mathrm{GeV}]$ & $N_{\text {events }}$ & $\mathscr{\mathscr { C }}\left[\mathrm{nb}^{-1}\right]$ & \multicolumn{1}{c|}{$\sigma_{\text {had }}[\mathrm{nb}]$} \\
\hline 91.254 & 155192 & 5124.9 & $30.45 \pm 0.10$ \\
\hline 88.480 & 4051 & 781.9 & $5.22 \pm 0.09$ \\
89.470 & 8531 & 846.7 & $10.15 \pm 0.12$ \\
90.228 & 14341 & 793.3 & $18.21 \pm 0.18$ \\
91.222 & 90686 & 3010.8 & $30.33 \pm 0.13$ \\
91.967 & 16070 & 657.6 & $24.64 \pm 0.24$ \\
92.966 & 10869 & 758.2 & $14.44 \pm 0.16$ \\
93.716 & 7953 & 793.6 & $10.10 \pm 0.13$ \\
\hline Totals & 307693 & 12783.0 & \\
\hline Systematic uncertainty & & $\pm 0.15 \%$ \\
\hline
\end{tabular}

\begin{tabular}{|c|r|r|r|}
\hline \multicolumn{4}{|c|}{1992 Data } \\
\hline$\sqrt{s}[\mathrm{GeV}]$ & $N_{\text {events }}$ & $\mathscr{L}\left[\mathrm{nb}^{-1}\right]$ & $\sigma_{\text {had }}[\mathrm{nb}]$ \\
\hline 91.294 & 677596 & 22424.7 & $30.451 \pm 0.047$ \\
\hline Systematic uncertainty & $\pm 0.15 \%$ \\
\hline
\end{tabular}

Table 10. Cross sections for $e^{+} e^{-} \rightarrow \mu^{+} \mu^{-}(\gamma)$, extrapolated to the full solid angle. The quoted systematic error excludes the uncertainty of $0.6 \%$ in the luminosity

\begin{tabular}{|c|c|c|c|}
\hline \multicolumn{4}{|c|}{1990 Data } \\
\hline$\sqrt{s}[\mathrm{GeV}]$ & $N_{\text {events }}$ & $\mathscr{C}\left[\mathrm{nb}^{-1}\right]$ & $\sigma_{\mu}[\mathrm{nb}]$ \\
\hline 88.231 & 66 & 388.6 & $0.268 \pm 0.033$ \\
\hline 89.236 & 104 & 421.0 & $0.388 \pm 0.038$ \\
\hline 90.238 & 217 & 364.9 & $0.931 \pm 0.063$ \\
\hline 91.230 & 2675 & 2822.4 & $1.478 \pm 0.028$ \\
\hline 92.226 & 282 & 394.8 & $1.116 \pm 0.066$ \\
\hline 93.228 & 160 & 496.6 & $0.506 \pm 0.040$ \\
\hline 94.223 & 123 & 480.4 & $0.405 \pm 0.036$ \\
\hline Totals & 3627 & 5368.7 & \\
\hline Systematic & ertainty & & $\pm 0.8 \%$ \\
\hline
\end{tabular}

\begin{tabular}{|c|r|r|c|}
\hline \multicolumn{4}{|c|}{ 1991 Data } \\
\hline$\sqrt{s}[\mathrm{GeV}]$ & $N_{\text {events }}$ & $\mathscr{C}\left[\mathrm{nb}^{-1}\right]$ & $\sigma_{\mu}[\mathrm{nb}]$ \\
\hline 91.254 & 5182 & 5039.5 & $1.510 \pm 0.021$ \\
\hline 88.480 & 135 & 779.4 & $0.259 \pm 0.022$ \\
89.470 & 280 & 849.9 & $0.486 \pm 0.029$ \\
90.228 & 471 & 793.2 & $0.871 \pm 0.040$ \\
91.222 & 2778 & 2925.6 & $1.393 \pm 0.026$ \\
91.967 & 567 & 699.9 & $1.190 \pm 0.050$ \\
92.966 & 368 & 758.2 & $0.718 \pm 0.037$ \\
93.716 & 267 & 829.8 & $0.478 \pm 0.029$ \\
\hline Totals & 9547 & 12675.5 & \\
\hline \multicolumn{5}{|c|}{ Systematic uncertainty } & $\pm 0.5 \%$ \\
\hline
\end{tabular}

\begin{tabular}{|c|c|c|c|}
\hline \multicolumn{4}{|c|}{1992 Data } \\
\hline$\sqrt{s}[\mathrm{GeV}]$ & $N_{\text {events }}$ & $\mathscr{C}\left[\mathrm{nb}^{-1}\right]$ & $\sigma_{\mu}[\mathrm{nb}]$ \\
\hline 91.294 & 20752 & 20748.5 & $1.466 \pm 0.010$ \\
\hline Systematic uncertainty & $\pm 0.5 \%$ \\
\hline
\end{tabular}

Table 11. Cross sections for $e^{+} e^{-} \rightarrow \tau^{+} \tau^{-}(\gamma)$, extrapolated to the full solid angle. The quoted systematic error excludes the uncertainty of $0.6 \%$ in the luminosity

\begin{tabular}{|c|c|c|c|}
\hline \multicolumn{4}{|c|}{1990 Data } \\
\hline $\begin{array}{ll}\sqrt{s} & {[\mathrm{GeV}]}\end{array}$ & $N_{\text {events }}$ & $\mathscr{E}\left[\mathrm{nb}^{-1}\right]$ & $\begin{array}{l}\sigma_{\tau}[\mathrm{nb}] \\
\end{array}$ \\
\hline 88.231 & 36 & 337.4 & $0.219 \pm 0.036$ \\
\hline 89.236 & 86 & 404.2 & $0.445 \pm 0.048$ \\
\hline 90.238 & 138 & 319.4 & $0.912 \pm 0.078$ \\
\hline 91.230 & 1887 & 2717.7 & $1.472 \pm 0.034$ \\
\hline 92.226 & 190 & 365.9 & $1.097 \pm 0.080$ \\
\hline 93.228 & 133 & 471.7 & $0.592 \pm 0.051$ \\
\hline 94.223 & 94 & 476.7 & $0.411 \pm 0.042$ \\
\hline Totals & 2564 & 5093.0 & \\
\hline \multicolumn{3}{|c|}{ Systematic uncertainty } & $\pm 0.9 \%$ \\
\hline
\end{tabular}

\begin{tabular}{|c|r|r|c|}
\hline \multicolumn{5}{|c|}{ 1991 Data } \\
\hline$\sqrt{s}[\mathrm{GeV}]$ & $N_{\text {events }}$ & $\mathscr{L}\left[\mathrm{nb}^{-1}\right]$ & $\sigma_{\tau}[\mathrm{nb}]$ \\
\hline 91.254 & 3720 & 4902.6 & $1.507 \pm 0.025$ \\
\hline 88.480 & 95 & 779.4 & $0.236 \pm 0.024$ \\
89.470 & 229 & 850.0 & $0.532 \pm 0.035$ \\
90.228 & 359 & 793.3 & $0.886 \pm 0.047$ \\
91.222 & 2102 & 2882.3 & $1.449 \pm 0.032$ \\
91.967 & 425 & 689.3 & $1.226 \pm 0.059$ \\
92.966 & 248 & 758.2 & $0.642 \pm 0.041$ \\
93.716 & 225 & 829.9 & $0.535 \pm 0.036$ \\
\hline Totals & 7403 & 12484.0 & \\
\hline Systematic uncertainty & & $\pm 0.7 \%$ \\
\hline
\end{tabular}

\begin{tabular}{|c|c|c|c|}
\hline \multicolumn{4}{|c|}{1992 Data } \\
\hline$\sqrt{s}[\mathrm{GeV}]$ & $N_{\text {events }}$ & $\mathscr{C}\left[\mathrm{nb}^{-1}\right]$ & $\sigma_{\tau}[\mathrm{nb}]$ \\
\hline 91.294 & 15300 & 20327.4 & $1.472 \pm 0.012$ \\
\hline Systematic uncertainty & $\pm 0.7 \%$ \\
\hline
\end{tabular}

Table 12. Cross sections for $e^{+} e^{-} \rightarrow e^{+} e^{-}(\gamma) . \sigma_{\mathrm{e}}$ is the efficiency corrected cross section for both leptons inside the angular range $44^{\circ}<\theta<$ $136^{\circ}$ with an acollinearity angle cut of $\xi<25^{\circ} . \sigma_{\mathrm{e}}^{s}$ is the $s$-channel contribution to the cross section extrapolated to the full solid angle (no acollinearity cut), see text. The quoted systematic error excludes the uncertainty of $0.6 \%$ in the luminosity

\begin{tabular}{|c|r|r|c|c|}
\hline \multicolumn{7}{|c|}{1990 Data } \\
\hline$\sqrt{s}[\mathrm{GeV}]$ & $N_{\text {events }}$ & $\mathscr{C}\left[\mathrm{nb}^{-1}\right]$ & $\sigma_{\mathrm{e}}[\mathrm{nb}]$ & $\sigma_{\mathrm{e}}^{\mathrm{s}}[\mathrm{nb}]$ \\
\hline 88.231 & 120 & 380.1 & $0.334 \pm 0.030$ & $0.186 \pm 0.052$ \\
89.236 & 237 & 466.3 & $0.533 \pm 0.034$ & $0.472 \pm 0.057$ \\
90.238 & 310 & 359.3 & $0.896 \pm 0.050$ & $1.037 \pm 0.082$ \\
91.230 & 3020 & 2960.9 & $1.053 \pm 0.019$ & $1.469 \pm 0.031$ \\
92.226 & 276 & 397.4 & $0.716 \pm 0.043$ & $1.137 \pm 0.070$ \\
93.228 & 198 & 505.5 & $0.406 \pm 0.029$ & $0.659 \pm 0.048$ \\
94.223 & 104 & 485.7 & $0.223 \pm 0.022$ & $0.344 \pm 0.037$ \\
\hline \multicolumn{7}{|c|}{ Totals } & 4265 & 5555.2 & \multicolumn{3}{c|}{} \\
\hline Systematic uncertainty & & $\pm 0.4 \%$ & $\pm 0.5 \%$ \\
\hline
\end{tabular}

\begin{tabular}{|c|r|r|c|c|}
\hline \multicolumn{5}{|c|}{ 1991 Data } \\
\hline$\sqrt{s}[\mathrm{GeV}]$ & $N_{\text {events }}$ & $\mathscr{B}\left[\mathrm{nb}^{-1}\right]$ & $\sigma_{\mathrm{e}}[\mathrm{nb}]$ & $\sigma_{\mathrm{e}}^{\mathrm{s}}[\mathrm{nb}]$ \\
\hline 91.254 & 5626 & 5548.4 & $1.033 \pm 0.014$ & $1.444 \pm 0.023$ \\
\hline 88.480 & 312 & 781.9 & $0.405 \pm 0.023$ & $0.297 \pm 0.040$ \\
89.470 & 487 & 860.4 & $0.574 \pm 0.026$ & $0.528 \pm 0.043$ \\
90.228 & 620 & 793.3 & $0.794 \pm 0.032$ & $0.869 \pm 0.052$ \\
91.222 & 3222 & 3076.2 & $1.069 \pm 0.019$ & $1.492 \pm 0.031$ \\
91.967 & 580 & 735.7 & $0.800 \pm 0.033$ & $1.242 \pm 0.054$ \\
92.966 & 316 & 758.2 & $0.423 \pm 0.024$ & $0.687 \pm 0.040$ \\
93.716 & 248 & 831.6 & $0.304 \pm 0.019$ & $0.486 \pm 0.032$ \\
\hline \multicolumn{7}{|c|}{ Totals } & 11351 & 13089.6 & \multicolumn{3}{|c|}{} \\
\hline Systematic uncertainty & & $\pm 0.3 \%$ & $\pm 0.4 \%$ \\
\hline
\end{tabular}

\begin{tabular}{|c|r|r|r|r|}
\hline \multicolumn{5}{|c|}{1992 Data } \\
\hline$\sqrt{s}[\mathrm{GeV}]$ & $N_{\text {events }}$ & $\mathscr{L}\left[\mathrm{nb}^{-\mathrm{T}}\right]$ & $\sigma_{\mathrm{e}}[\mathrm{nb}]$ & $\sigma_{\mathrm{e}}^{\mathrm{s}}[\mathrm{nb}]$ \\
\hline 91.294 & 22726 & 22189.4 & $1.055 \pm 0.007$ & $1.491 \pm 0.011$ \\
\hline Systematic uncertainty & & $\pm 0.3 \%$ & $\pm 0.4 \%$ \\
\hline
\end{tabular}


Table 13. Forward-backward asymmetries, $A_{\mathrm{fb}}^{\ell}$, for $e^{+} e^{-} \rightarrow \ell^{+} \ell^{-}(\gamma), \ell=\mu, \tau, \mathrm{e}$, including a cut on the acollinearity angle, $\xi<15^{\circ}$ for muons, $\xi<14.3^{\circ}$ for taus, and $\xi<25^{\circ}$ for electrons. $A_{\mathrm{fb}}^{\mathrm{e}}$ is the asymmetry determined from counting in the angular range of $44^{\circ}<\theta<136^{\circ}$ for both leptons. $A_{\mathrm{fb}}^{\mathrm{e}, \mathrm{s}}$ is the $s$-channel contribution to the forward-backward asymmetry extrapolated to the full solid angle $\left(\xi<25^{\circ}\right)$, see text

\begin{tabular}{|c|r|r|r|r|}
\hline \multicolumn{5}{|c|}{1990 Data } \\
\hline$\sqrt{s}[\mathrm{GeV}]$ & \multicolumn{1}{|c|}{$A_{\mathrm{fb}}^{\mu}$} & \multicolumn{1}{c|}{$A_{\mathrm{fh}}^{\tau}$} & \multicolumn{1}{c|}{$A_{\mathrm{fh}}^{\mathrm{e}}$} & \multicolumn{1}{c|}{$A_{\mathrm{fh}}^{\mathrm{es}}$} \\
\hline 88.231 & $-0.391 \pm 0.117$ & $-0.36 \pm 0.20$ & $0.520 \pm 0.095$ & $-0.141 \pm 0.344$ \\
89.236 & $-0.044 \pm 0.109$ & $0.00 \pm 0.15$ & $0.296 \pm 0.070$ & $-0.193 \pm 0.136$ \\
90.238 & $-0.184 \pm 0.074$ & $-0.13 \pm 0.11$ & $0.155 \pm 0.064$ & $-0.132 \pm 0.091$ \\
91.230 & $0.006 \pm 0.021$ & $0.077 \pm 0.028$ & $0.101 \pm 0.021$ & $-0.014 \pm 0.025$ \\
92.226 & $0.110 \pm 0.066$ & $0.09 \pm 0.09$ & $0.040 \pm 0.069$ & $0.033 \pm 0.071$ \\
93.228 & $0.095 \pm 0.091$ & $0.07 \pm 0.11$ & $0.083 \pm 0.081$ & $0.092 \pm 0.082$ \\
94.223 & $0.134 \pm 0.099$ & $0.04 \pm 0.13$ & $0.144 \pm 0.118$ & $0.112 \pm 0.129$ \\
\hline Systematic uncertainty & \pm 0.005 & \pm 0.005 & \pm 0.004 & \pm 0.005 \\
\hline
\end{tabular}

\begin{tabular}{|c|r|r|r|r|}
\hline \multicolumn{5}{|c|}{1991 Data } \\
\hline$\sqrt{s}[\mathrm{GeV}]$ & \multicolumn{1}{|c|}{$A_{\mathrm{fb}}^{\mu}$} & \multicolumn{1}{c|}{$A_{\mathrm{fb}}^{\tau}$} & \multicolumn{1}{c|}{$A_{\mathrm{fb}}^{\mathrm{e}}$} & \multicolumn{1}{c|}{$A_{\mathrm{fh}}^{e, s}$} \\
\hline 91.254 & $0.028 \pm 0.014$ & $0.037 \pm 0.021$ & $0.110 \pm 0.016$ & $-0.002 \pm 0.019$ \\
\hline 88.480 & $-0.197 \pm 0.097$ & $-0.106 \pm 0.128$ & $0.384 \pm 0.063$ & $-0.232 \pm 0.156$ \\
89.470 & $-0.191 \pm 0.063$ & $-0.152 \pm 0.083$ & $0.333 \pm 0.051$ & $-0.081 \pm 0.098$ \\
90.228 & $-0.101 \pm 0.050$ & $-0.137 \pm 0.070$ & $0.253 \pm 0.046$ & $-0.037 \pm 0.071$ \\
91.222 & $-0.002 \pm 0.020$ & $-0.032 \pm 0.029$ & $0.125 \pm 0.022$ & $0.014 \pm 0.026$ \\
91.967 & $0.058 \pm 0.043$ & $0.042 \pm 0.063$ & $0.167 \pm 0.048$ & $0.147 \pm 0.051$ \\
92.966 & $0.117 \pm 0.056$ & $0.161 \pm 0.080$ & $0.070 \pm 0.066$ & $0.080 \pm 0.067$ \\
93.716 & $0.089 \pm 0.065$ & $0.058 \pm 0.082$ & $0.150 \pm 0.074$ & $0.147 \pm 0.077$ \\
\hline Systematic uncertainty & \pm 0.002 & \pm 0.005 & \pm 0.004 & \pm 0.005 \\
\hline
\end{tabular}

\begin{tabular}{|c|r|r|r|r|}
\hline \multicolumn{5}{|c|}{1992 Data } \\
\hline$\sqrt{s}[\mathrm{GeV}]$ & \multicolumn{1}{|c|}{$A_{\mathrm{fh}}^{\mu}$} & \multicolumn{1}{|c|}{$A_{\mathrm{fh}}^{\tau}$} & \multicolumn{1}{c|}{$A_{\mathrm{fh}}^{\mathrm{e}}$} & \multicolumn{1}{c|}{$A_{\mathrm{fb}}^{\mathrm{es}}$} \\
\hline 91.294 & $0.007 \pm 0.007$ & $0.015 \pm 0.010$ & $0.104 \pm 0.007$ & $0.001 \pm 0.008$ \\
\hline Systematic uncertainty & \pm 0.002 & \pm 0.003 & \pm 0.002 & \pm 0.003 \\
\hline
\end{tabular}

\section{Determination of electroweak parameters}

$$
\begin{aligned}
\sigma_{\mathrm{e}}^{\mathrm{s}} & =\left[\sigma_{\mathrm{e}}-\sigma_{\mathrm{e}}^{\mathrm{l}+\mathrm{s} / \mathrm{t}}\left(44^{\circ}<\theta<136^{\circ}, \xi<25^{\circ}\right)_{\mathrm{SM}}\right] \\
\cdot & \left(\frac{\sigma_{\mathrm{e}}^{\mathrm{s}}}{\sigma_{\mathrm{e}}^{\mathrm{s}}\left(44^{\circ}<\theta<136^{\circ}, \xi<25^{\circ}\right)}\right)_{\mathrm{SM}} \\
\delta \sigma_{\mathrm{e}}^{\mathrm{s}} & =\delta \sigma_{\mathrm{e}} \cdot\left(\frac{\sigma_{\mathrm{e}}^{\mathrm{s}}}{\sigma_{\mathrm{e}}^{\mathrm{s}}\left(44^{\circ}<\theta<136^{\circ}, \xi<25^{\circ}\right)}\right)_{\mathrm{SM}} .
\end{aligned}
$$

In the case of the forward-backward asymmetry, $A_{\mathrm{fb}}=$ $\left(\sigma_{\mathrm{f}}-\sigma_{\mathrm{b}}\right) /\left(\sigma_{\mathrm{f}}+\sigma_{\mathrm{b}}\right)$, we perform the analogous calculation for $\sigma_{\mathrm{f}}$ and $\sigma_{\mathrm{b}}$. The extrapolated s-channel cross sections, $\sigma_{\mathrm{e}}^{\mathrm{s}}$, without any cuts, and s-channel asymmetries, $A_{\mathrm{fb}}^{\mathrm{e}, \mathrm{s}}$, with an acollinearity angle cut of $\xi<25^{\circ}$, are also given (Tables 12 and 13). The additional systematic errors introduced by this procedure are estimated to be $0.2 \%$ in the case of cross sections and 0.002 in the case of asymmetries. They are given by the uncertainties in the corrections due to the allowed range of values for the SM parameters used for the ALIBABA calculations, $m_{Z}=91.195 \pm 0.009 \mathrm{GeV}$, $\alpha_{s}=0.123 \pm 0.006$ [4], and taking $m_{t}=150 \pm 50 \mathrm{Gev}$, $m_{\mathrm{H}}=300_{-240}^{+700} \mathrm{GeV}$.

All results are corrected for the $51 \mathrm{MeV}$ spread in the center-of-mass energy, which is due to the finite spread of the particle energy in the LEP beams [22]. The correction is largest for cross sections on the peak, where it increases the observed cross section by $0.14 \%$. The split of the 1991 data sample taken at the peak energy is introduced because the accuracy in the calibration of the LEP beam energy changed during the 1991 run [22].
Three different approaches are used to extract the electroweak parameters from the measured total cross sections and forward-backward asymmetries.

The first two approaches determine the electroweak parameters making a minimum of assumptions about any underlying theory, for example the Standard Model (SM). The first approach uses only the total cross-section data to determine the parameters of the $Z$ boson, its mass, $m_{\mathrm{Z}}$, total decay width, $\Gamma_{Z}$, and partial decay width to fermion pairs $(f \bar{f}), \Gamma_{f}$. The second approach also includes the asymmetry data, which allows the determination of the coupling constants of the neutral weak current. The third approach evaluates all the data within the framework of the SM in order to determine the input parameters of the SM, such as the mass of the top quark.

In all three approaches, a Breit-Wigner ansatz is used to describe the $\mathrm{Z}$ boson. The mass, $m_{Z}$, and the total width, $\Gamma_{Z}$, of the $Z$ boson are defined by the functional form of the Breit-Wigner denominator, which explicitly takes into account the energy dependence of the total width. The total cross section to lowest order, $\sigma^{\circ}$, for the process $e^{+} e^{-} \rightarrow f \bar{f}$, $f \neq e$, is given by the sum of three terms, the $Z$ exchange, $\sigma_{\mathrm{Z}}^{\circ}$, the photon exchange, $\sigma_{\gamma}^{\circ}$, and the $\gamma Z$ interference, $\sigma_{\mathrm{int}}^{\circ}$ :

$$
\begin{aligned}
\sigma^{\circ} & =\sigma_{\mathrm{Z}}^{\circ}+\sigma_{\gamma}^{\circ}+\sigma_{\mathrm{int}}^{\circ} \\
\sigma_{\mathrm{Z}}^{\circ} & =\frac{12 \pi}{m_{\mathrm{Z}}^{2}} \frac{\Gamma_{\mathrm{e}} \Gamma_{f}}{\Gamma_{Z}^{2}} \frac{s \Gamma_{Z}^{2}}{\left(s-m_{\mathrm{Z}}^{2}\right)^{2}+s^{2} \Gamma_{Z}^{2} / m_{\mathrm{Z}}^{2}} \\
\sigma_{\gamma}^{\circ} & =\frac{4 \pi \alpha^{2}}{3 s} q_{e}^{2} q_{f}^{2} N_{C}^{f}
\end{aligned}
$$


$\sigma_{\mathrm{int}}^{\circ}=\frac{4 \pi \alpha^{2}}{3} J_{f} \frac{s-m_{\mathrm{Z}}^{2}}{\left(s-m_{\mathrm{Z}}^{2}\right)^{2}+s^{2} \Gamma_{Z}^{2} / m_{\mathrm{Z}}^{2}}$

where $q_{f}$ is the electric charge of the final-state fermion, $N_{C}^{f}$ its color factor, and $\alpha$ the electromagnetic coupling constant. The pure photon exchange is determined by QED.

The first two approaches treat the mass and the total and partial widths of the $Z$ boson as free and independent parameters. The interference of the $Z$ exchange with the photon exchange adds another parameter, the $\gamma Z$-interference term, $J_{f}$, besides those corresponding to mass and widths of the Z. Since in the SM $\left|\sigma_{\text {int }}^{\circ}(s)\right| \ll \sigma^{\circ}(s)$ for center-of-mass energies, $\sqrt{s}$, close to $m_{\mathrm{Z}}$, it is difficult to measure $J_{f}$ accurately at current LEP energies [23]. The $\gamma Z$-interference term is usually taken from the SM [3-5, 24, 25], thus making assumptions about the form of the electroweak unification. If instead the $\gamma Z$-interference term is determined from the data, the errors on correlated parameters, such as the mass of the $Z$ boson, are increased [23].

The second approach determines the vector and axialvector coupling constants of the neutral weak current to charged leptons, $g_{V}^{\ell}$ and $g_{A}^{\ell}$, by using the forward-backward asymmetries in addition to the total cross sections. For center-of-mass energies close to $m_{\mathrm{Z}}$, the forward-backward asymmetry to lowest order, $A_{\mathrm{fb}}^{\circ, \ell}$, for the process $e^{+} e^{-} \rightarrow$ $\ell^{+} \ell^{-}, \ell \neq e$, is given by:

$$
\begin{aligned}
A_{\mathrm{fb}}^{\circ, \ell}(s) & =\frac{3}{4} A_{e} A_{\ell}\left[1+q_{e} q_{\ell}\left(\frac{1}{g_{V}^{e} g_{V}^{\ell}}-\frac{A_{e} A_{\ell}}{g_{A}^{e} g_{A}^{\ell}}\right)\right. \\
& \left.\times \frac{\sqrt{2} \pi \alpha}{G_{F} m_{Z}^{2}} \cdot \frac{s-m_{\mathrm{Z}}^{2}}{s}\right] \\
& +\odot\left(\frac{s-m_{\mathrm{Z}}^{2}}{s}\right)^{2} \\
A_{\ell} & =\frac{2 g_{V}^{\ell} g_{A}^{\ell}}{\left(g_{V}^{\ell}\right)^{2}+\left(g_{A}^{\ell}\right)^{2}} .
\end{aligned}
$$

In (2) and (4), the leptonic partial width, $\Gamma_{\ell}$, and the leptonic $\gamma Z$-interference term, $J_{\ell}$, are now expressed in terms of $g_{V}^{\ell}$ and $g_{A}^{\ell}$ :

$\Gamma_{\ell}=\frac{G_{F} m_{\mathrm{Z}}^{3}}{6 \sqrt{2} \pi}\left[\left(g_{V}^{\ell}\right)^{2}+\left(g_{A}^{\ell}\right)^{2}\right]$

$J_{\ell}=\frac{G_{F} m_{\mathrm{Z}}^{2}}{\sqrt{2} \pi \alpha} q_{e} q_{\ell} g_{V}^{e} g_{V}^{\ell}$

where $G_{F}$ is the Fermi coupling constant. This approach cannot be applied to the hadronic final state, which is summed over all colors and open quark flavors. Therefore, the parameterization of the first approach is used to express the hadronic cross section in terms of $\Gamma_{\text {had }}$ and $J_{\text {had }}$.

The forward-backward asymmetry measures the vector and axial-vector coupling constants, $g_{V}^{\ell}$ and $g_{A}^{\ell}$, in a different combination than $\Gamma_{\ell}$. Its energy dependence distinguishes $g_{V}^{\ell}$ and $g_{A}^{\ell}$, see (5).

The QED radiative corrections on the total cross sections and forward-backward asymmetries are included by convolution and by the replacement $\alpha \rightarrow \alpha(s)=\alpha /(1-\Delta \alpha)$ to account for the running of the electromagnetic coupling constant [26, 27]. Weak corrections cannot be calculated without assumptions about the underlying electroweak theory, such as the mass of the top quark, $m_{t}$, and the mass of the Higgs boson, $m_{H}$, in the SM. Therefore, we define the effective coupling constants, $\bar{g}_{V}^{\ell}$ and $\bar{g}_{A}^{\ell}$, which absorb these weak corrections.

Assuming lepton universality, the effective couplings are expressed in terms of the effective electroweak mixing angle, $\sin ^{2} \bar{\theta}_{W}$, and the effective ratio of the neutral to charged weak current couplings, $\bar{\rho}=1 /(1-\Delta \bar{\rho})[28]$ :

$\bar{g}_{V}^{\ell}=\sqrt{\bar{\rho}} \cdot\left(I_{3}^{\ell}-2 q_{\ell} \sin ^{2} \bar{\theta}_{W}\right)$

$\bar{g}_{A}^{\ell}=\sqrt{\bar{\rho}} \cdot I_{3}^{\ell}$,

where $I_{3}^{\ell}$ is the third component of the weak isospin of the lepton, $\ell$.

The third approach to determine electroweak parameters uses the framework of the SM. By comparing its predictions with the set of experimental measurements, it is possible to test the consistency of the SM.

The input parameters of the SM are $\alpha$, the fermion masses, $m_{H}, m_{\mathrm{Z}}$, and the mass of the $\mathrm{W}$ boson, $m_{W}$. QCD adds one more parameter, the strong coupling constant, $\alpha_{s}$. The electromagnetic coupling constant and the fermion masses, with the exception of the top-quark mass, are known with sufficient precision. The effect on the radiative corrections due to the mass of the Higgs boson is too small to be measurable. While the $Z$ mass is measured with high precision at LEP, the mass of the $\mathrm{W}$ boson is not known with similar precision. Therefore, $m_{W}$ is replaced by the Fermi coupling constant, $G_{F}$, measured in muon decay, using the relation [29]:

$$
\frac{G_{F}}{\sqrt{2}}=\frac{\pi \alpha}{2} \cdot \frac{1}{m_{\mathrm{Z}}^{2} \sin ^{2} \theta_{W} \cos ^{2} \theta_{W}} \cdot \frac{1}{1-\Delta r},
$$

where $\sin ^{2} \theta_{W}$ is defined as:

$\sin ^{2} \theta_{W}=1-\frac{m_{W}^{2}}{m_{\mathrm{Z}}^{2}}$

and $\Delta r$ takes into account the electroweak radiative corrections, $\Delta r=\Delta \alpha-\cot ^{2} \theta_{W} \Delta \bar{\rho}+\Delta r_{\text {remainder }}$ [29]. With this procedure the relevant unknown parameters of the SM are $m_{\mathrm{Z}}, \alpha_{\mathrm{s}}$ and $m_{t}$.

The results of the three approaches are given in the subsections below.

In all three analysis procedures we use the analytical program ZFITTER 4.60 [19] for the calculation of the higherorder corrections and the predictions of the SM. ZFITTER includes electroweak radiative corrections to $\mathscr{O}(\alpha)$ and a common exponentiation of initial- and final-state bremsstrahlung. The corrections to $\mathscr{Q}\left(\alpha^{2}\right)$ are taken into account in the leading-log approximation and include the production of photon and fermion pairs in the initial state. Furthermore, the $\mathscr{O}(\alpha)$ and $\mathscr{O}\left(\alpha^{2}\right)$ corrections are supplemented with the $\mathscr{O}\left(\alpha \alpha_{s} m_{t}^{2} / m_{W}^{2}\right)$ and the $\mathscr{Q}\left(\alpha^{2} m_{t}^{4} / m_{W}^{4}\right)$ corrections from top-quark insertions in the gauge-boson self energies and in the $Z b \bar{b}$ vertex. In the case of the b-quark asymmetries, the $\mathscr{Q}\left(\alpha \alpha_{s} m_{t}^{2} / m_{W}^{2}\right)$ corrections of the $Z b \bar{b}$ vertex are not included as they are not yet available. QCD corrections in final states with quarks are considered up to Q $\left(\alpha_{s}^{3}\right)$. 
The electroweak parameters are determined in a $\chi^{2}$ fit using the MINUIT program [30]. The $\chi^{2}$ is constructed from the measurements, their errors including the correlations, and the theoretical expectations.

In the case of the process $e^{+} e^{-} \rightarrow e^{+} e^{-}(\gamma)$, the existence of the t-channel exchange of the $\gamma$ and $Z$ bosons and its interference with the s-channel exchange lead to additional complications. Analytical programs to calculate this process, such as the program ALIBABA [21], are not directly suited for fitting purposes, as computationally they are very time consuming. Thus, the following procedure is adopted. During the initialization of a fit, ALIBABA is used once to calculate the predictions of the $t$-channel and $s / t$-interference contributions to the measured $e^{+} e^{-} \rightarrow e^{+} e^{-}(\gamma)$ cross sections and forward-backward asymmetries. ZFITTER is used during the fits to calculate the corresponding s-channel contributions as a function of the varying electroweak parameters. Since the $\mathrm{t}$-channel and $\mathrm{s} / \mathrm{t}$-interference contributions also depend to some extent on the fitted parameters such as $m_{\mathrm{Z}}$, the fits are iterated. This procedure converges after two iterations. The systematic error introduced by this treatment is included in the total error.

In addition to the experimental errors, we take into account the uncertainties in the determination of the LEP center-of-mass energy [22]. The dominant systematic error on $m_{\mathrm{Z}}$ arising from the LEP energy calibration is due to the error on the absolute energy scale, which for the 1990 data is $26 \mathrm{MeV}$, for the 1991 data before 14 August is $18 \mathrm{MeV}$ and $5.3 \mathrm{MeV}$ thereafter, and for the 1992 data is $18 \mathrm{MeV}$. This leads to a systematic error of $7 \mathrm{MeV}$ on the $Z$ mass. The systematic error on $\Gamma_{Z}$ due to the LEP energy calibration is $5 \mathrm{MeV}$, which is dominated by the uncertainty in the relative energy scale.

Further details about the fitting procedures can be found in [4].

\subsection{Properties of the $Z$ boson}

Using the first approach described above, we carry out fits to the total cross-section data of the reactions $e^{+} e^{-} \rightarrow$ hadrons, $e^{+} e^{-}, \mu^{+} \mu^{-}$and $\tau^{+} \tau^{-}$to determine the mass and the total and partial widths of $Z$ boson. The results are summarized Table 14 . For the mass, $m_{\mathrm{Z}}$, and the total width, $\Gamma_{Z}$, we find:

$$
\begin{aligned}
M_{\mathrm{Z}} & =91195 \pm 6 \pm 7(\text { LEP }) \mathrm{MeV} \\
\Gamma_{\mathrm{Z}} & =2494 \pm 9 \pm 5(\text { LEP }) \mathrm{MeV} .
\end{aligned}
$$

The first error is experimental, and the second error arises from the uncertainties in the LEP energy calibration. In this fit, the $\gamma Z$-interference term, $J_{f}$, is fixed to its $S M$ value, which leads to an error of less than $1 \mathrm{MeV}$ on $m_{\mathrm{Z}}$ due to the SM dependence of $J_{f}$ on $m_{t}$ and $m_{H}$.

If we instead leave the leptonic and hadronic $\gamma Z$-interference terms as free parameters to be determined from the data, we obtain:

$M_{\mathrm{Z}}=91190 \pm 6 \pm 7 \pm 9\left(J_{\text {had }}\right) \mathrm{MeV}$.

The correlation between the mass of the $\mathrm{Z}, m_{\mathrm{Z}}$, and the hadronic $\gamma Z$-interference term, $J_{\text {had }}$, is shown in Fig. 12. In
Table 14. Results on the mass and total and partial widths of the $Z$ boson derived from the cross-section data. SM expectations are listed using the fitted $Z$ mass, $m_{Z}=91.195 \pm 0.009 \mathrm{GeV}, \alpha_{\mathrm{s}}=0.123 \pm 0.06$ and taking $m_{t}=150 \pm 50 \mathrm{GeV}, m_{H}=300_{-240}^{+700} \mathrm{GeV}$

\begin{tabular}{|c|c|c|c|}
\hline $\begin{array}{c}\text { Parameters } \\
{[\mathrm{MeV}]}\end{array}$ & \multicolumn{2}{|c|}{ Treatment of charged leptons } & Standard \\
& Non-universality & Universality & Model \\
\hline$m_{\mathrm{Z}}$ & $91195 \pm 6 \pm 7$ (LEP) & $91195 \pm 6 \pm 7$ (LEP) & - \\
$\Gamma_{Z}$ & $2494 \pm 9 \pm 5$ (LEP) & $2494 \pm 9 \pm 5$ (LEP) & $2491_{-18}^{+21}$ \\
$\Gamma_{\mathrm{had}}$ & $1749 \pm 11$ & $1748 \pm 10$ & $1739_{-14}^{+16}$ \\
\hline$\Gamma_{e}$ & $83.43 \pm 0.52$ & - & \\
$\Gamma_{\mu}$ & $83.20 \pm 0.79$ & - & \\
$\Gamma_{\tau}$ & $84.04 \pm 0.94$ & - & \\
$\Gamma_{\ell}$ & - & $83.49 \pm 0.46$ & $83.7_{-0.5}^{+0.6}$ \\
\hline$\chi^{2} / \mathrm{DOF}$ & $52 / 58$ & $53 / 60$ & - \\
\hline
\end{tabular}

such a fit, the uncertainty on the $\gamma Z$-interference term substantially increases the total error on $m_{Z}$ from 9 to $13 \mathrm{MeV}$. The errors on the total and partial widths of the $Z$ boson increase only marginally. In order to reduce this additional uncertainty on $m_{\mathrm{Z}}$, a better determination of $J_{f}$ is necessary, which can be achieved by accurately measuring cross sections further away from the $Z$ resonance peak [31, 32].

If we do not assume universality of charged leptons, we obtain the partial decay widths of the $Z$ boson to the three types of charged leptons separately. The three partial widths are in good agreement with each other (Table 14). Assuming lepton universality, we fit for one leptonic partial width, $\Gamma_{\ell}$, instead of three, where $\Gamma_{\ell}$ is defined as the partial decay width of the $Z$ into a pair of massless charged leptons. The partial decay widths for the inclusive hadronic and charged leptonic final states are given by:

$$
\begin{aligned}
\Gamma_{\text {had }} & =1748 \pm 10 \mathrm{MeV} \\
\Gamma_{\ell} & =83.49 \pm 0.46 \mathrm{MeV} .
\end{aligned}
$$

Our results on the total and partial widths of the $Z$ boson are in good agreement with the predictions of the SM.

From the total and partial widths and their correlations, we derive the decay width of the $Z$ boson into invisible particles:

$\Gamma_{\mathrm{inv}}=\Gamma_{\mathrm{Z}}-\Gamma_{\mathrm{had}}-\left(3+\delta_{\tau}\right) \cdot \Gamma_{\ell}=496.5 \pm 7.9 \mathrm{MeV}$,

where $\delta_{\tau}=-0.0023$ includes the effect of the tau mass [28]. Within the SM, the invisible width is exclusively given by the decay of the $Z$ into neutrinos. Thus, $\Gamma_{\text {inv }}$ determines the number, $N_{\nu}$, of light neutrino species. In order to obtain a result independent of the unknown parameters of the SM, such as the top-quark and Higgs-boson masses, the following relation is used to evaluate $N_{\nu}$ :

$N_{\nu}=\frac{\Gamma_{\mathrm{inv}}}{\Gamma_{\ell}}\left(\frac{\Gamma_{\ell}}{\Gamma_{\nu}}\right)_{\mathrm{SM}}$.

Most higher-order corrections involving $m_{t}$ and $m_{H}$ cancel in the ratio:

$$
\left(\frac{\Gamma_{\ell}}{\Gamma_{\nu}}\right)_{\mathrm{SM}}=0.5015 \pm 0.0007
$$

where the error is due to residual $m_{t}$ and $m_{H}$ dependence estimated by varying $m_{t}$ between 100 and $200 \mathrm{GeV}$, and $m_{H}$ between 60 and $1000 \mathrm{GeV}$. The value of $N_{\nu}$ is determined to be: 
Table 15. Results on the peak hadronic cross section, $\sigma_{\text {had, }}^{\circ}$ and peak leptonic asymmetries, $A_{\mathrm{fb}}^{\circ, \ell}$, unfolded for QED corrections, and ratios of hadronic to leptonic widths, $R_{\text {had }}^{\ell}$, derived from the forward-backward asymmetry and cross-section data. SM expectations are listed using the fitted $Z$ mass, $m_{Z}=91.195 \pm 0.009 \mathrm{GeV}, \alpha_{s}=0.123 \pm 0.006$ and taking $m_{t}=150 \pm 50 \mathrm{GeV}, m_{H}=300_{-240}^{+700} \mathrm{GeV}$

\begin{tabular}{|c|c|c|c|}
\hline Parameter & \multicolumn{2}{|c|}{ Treatment of charged leptons } & Standard \\
& Non-universality & Universality & Model \\
\hline$m_{\mathrm{Z}}[\mathrm{MeV}]$ & $91195 \pm 6 \pm 7$ (LEP) & $91195 \pm 6 \pm 7$ (LEP) & - \\
$\Gamma_{Z}[\mathrm{MeV}]$ & $2495 \pm 9 \pm 5$ (LEP) & $2495 \pm 9 \pm 5$ (LEP) & $2491_{-18}^{+21}$ \\
$\sigma_{\mathrm{had}}^{\circ}[\mathrm{nb}]$ & $41.39 \pm 0.26$ & $41.42 \pm 0.26$ & $41.43_{-0.06}^{+0.08}$ \\
\hline$R_{\mathrm{had}}^{e}$ & $20.96 \pm 0.15$ & - & \\
$R_{\mathrm{had}}^{\mu}$ & $21.02 \pm 0.16$ & - & \\
$R_{\mathrm{had}}^{\tau}$ & $20.80 \pm 0.20$ & - & \\
$R_{\mathrm{had}}^{\ell}$ & - & $20.93 \pm 0.10$ & $20.77 \pm 0.07$ \\
\hline$A_{\mathrm{fb}}^{\ell, e}$ & $0.0104 \pm 0.0092$ & - & \\
$A_{\mathrm{fb}}^{\mathrm{o}, \mu}$ & $0.0179 \pm 0.0061$ & - & \\
$A_{\mathrm{fb}}^{\mathrm{o}, \tau}$ & $0.0265 \pm 0.0088$ & - & \\
$A_{\mathrm{fb}}^{\mathrm{o}, \ell}$ & - & $0.0184 \pm 0.0045$ & $0.014_{-0.003}^{+0.005}$ \\
\hline$\chi^{2 / D O F}$ & $84 / 103$ & $87 / 107$ & - \\
\hline
\end{tabular}

Table 16. Correlation matrix of the parameters $m_{\mathrm{Z}}, \Gamma_{Z}, \sigma_{\mathrm{had}}^{\circ}, R_{\mathrm{had}}^{\ell}$ and $A_{\mathrm{fb}}^{\mathrm{o}, \ell}$, for $\ell=\mathrm{e}, \mu, \tau$, not assuming lepton universality

\begin{tabular}{|c|rrrrrrrrr|}
\hline Parameter & $M_{\mathrm{Z}}$ & $\Gamma_{\mathrm{Z}}$ & $\sigma_{\text {had }}^{\circ}$ & $R_{\mathrm{had}}^{e}$ & \multicolumn{1}{c|}{$R_{\text {had }}^{\mu}$} & $R_{\text {had }}^{\tau}$ & $A_{\mathrm{fb}}^{\circ, e}$ & \multicolumn{1}{c|}{$A_{\mathrm{fb}}^{\circ, \mu}$} & \multicolumn{1}{c|}{$A_{\mathrm{fb}}^{\circ, \tau}$} \\
\hline$M_{\mathrm{Z}}$ & 1.000 & 0.079 & -0.004 & 0.008 & 0.001 & 0.000 & 0.033 & 0.055 & 0.038 \\
$\Gamma_{\mathrm{Z}}$ & 0.079 & 1.000 & -0.106 & -0.011 & -0.006 & -0.002 & 0.004 & 0.000 & 0.001 \\
$\sigma_{\text {had }}^{\circ}$ & -0.004 & -0.106 & 1.000 & -0.045 & 0.069 & 0.055 & 0.088 & -0.009 & -0.006 \\
$R_{\text {had }}^{e}$ & 0.008 & -0.011 & -0.045 & 1.000 & 0.067 & 0.052 & -0.102 & 0.002 & 0.001 \\
$R_{\text {had }}^{\mu}$ & 0.001 & -0.006 & 0.069 & 0.067 & 1.000 & 0.050 & -0.001 & 0.012 & 0.000 \\
$R_{\text {had }}^{\tau}$ & 0.000 & -0.002 & 0.055 & 0.052 & 0.050 & 1.000 & -0.001 & 0.000 & 0.010 \\
$A_{\mathrm{fb}}^{\mathrm{O}, e}$ & 0.033 & 0.004 & 0.088 & -0.102 & -0.001 & -0.001 & 1.000 & 0.016 & 0.011 \\
$A_{\mathrm{fb}}^{\mathrm{o}, \mu}$ & 0.055 & 0.000 & -0.009 & 0.002 & 0.012 & 0.000 & 0.016 & 1.000 & 0.018 \\
$A_{\mathrm{fb}}^{\mathrm{O}, \tau}$ & 0.038 & 0.001 & -0.006 & 0.001 & 0.000 & 0.010 & 0.011 & 0.018 & 1.000 \\
\hline
\end{tabular}

Table 17. Correlation matrix of the parameters $m_{Z}, \Gamma_{Z}, \sigma_{\text {had }}^{\circ}, R_{\text {had }}^{\ell}$ and $A_{\mathrm{fb}}^{\circ, \ell}$, assuming lepton universality

\begin{tabular}{|c|rrrrr|}
\hline Parameter & $M_{\mathrm{Z}}$ & $\Gamma_{\mathrm{Z}}$ & $\sigma_{\mathrm{had}}^{\circ}$ & $R_{\mathrm{had}}^{\ell}$ & $A_{\mathrm{fb}}^{\circ, \ell}$ \\
\hline$M_{\mathrm{Z}}$ & 1.000 & 0.079 & -0.003 & 0.006 & 0.074 \\
$\Gamma_{\mathrm{Z}}$ & 0.079 & 1.000 & -0.108 & -0.010 & 0.002 \\
$\sigma_{\mathrm{had}}^{\mathrm{o}}$ & -0.003 & -0.108 & 1.000 & 0.043 & 0.028 \\
$R_{\mathrm{had}}^{\ell}$ & 0.006 & -0.010 & 0.043 & 1.000 & -0.020 \\
$A_{\mathrm{fb}}^{\circ, \ell}$ & 0.074 & 0.002 & 0.028 & -0.020 & 1.000 \\
\hline
\end{tabular}

$N_{\nu}=2.981 \pm 0.050$,

where the error is dominated by the error in the luminosity measurement. The above results are in agreement with the determination of $\Gamma_{\mathrm{inv}}$ derived from the measurement of the $e^{+} e^{-} \rightarrow \nu \bar{\nu} \gamma$ cross section [33].

\subsection{Coupling constants of the neutral weak current}

Fits to the hadronic cross-section and leptonic cross-section and forward-backward asymmetry data are performed using the second approach described above, with and without the assumption of lepton universality. Table 15 summarizes the results in terms of the hadronic cross section, $\sigma_{\text {had }}^{\circ}$, the leptonic asymmetries, $A_{\mathrm{fb}}^{\circ, \ell}$, at $s=m_{\mathrm{Z}}^{2}$ and unfolded for QED corrections, and the ratio of the hadronic to the leptonic partial widths, $R_{\text {had }}^{\ell}$ :

$\sigma_{\mathrm{had}}^{\circ}=\frac{12 \pi}{m_{\mathrm{Z}}^{2}} \cdot \frac{\Gamma_{e} \Gamma_{\mathrm{had}}}{\Gamma_{Z}^{2}}$

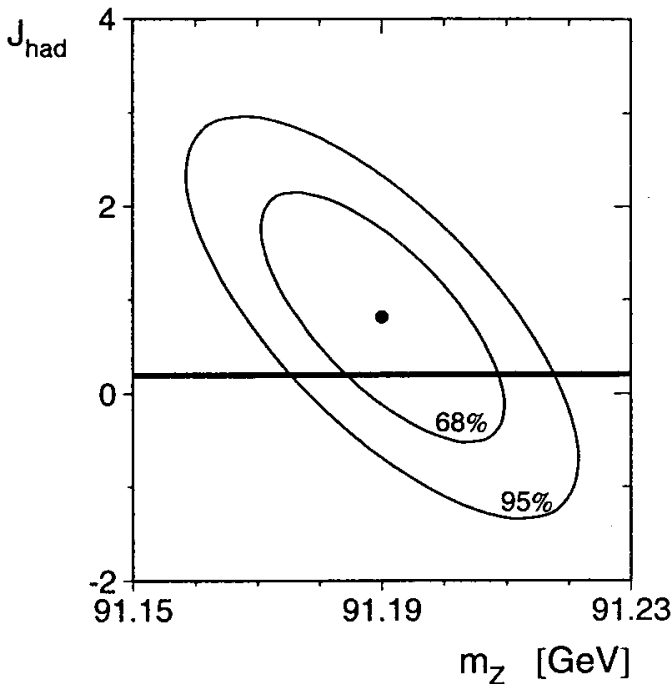

Fig. 12. The correlation between the mass of the $Z$ boson, $m_{\mathrm{Z}}$, and the hadronic $\gamma Z$-interference term, $J_{\text {had }}$. The $68 \%$ and $95 \%$ confidence-level contours for the two parameters are shown. The band shows the prediction for $J_{\text {had }}$ according to the SM, using $\alpha_{\mathrm{s}}=0.123 \pm 0.006$ and taking $m_{t}=$ $150 \pm 50 \mathrm{GeV}, m_{H}=300_{-240}^{+700} \mathrm{GeV}$

$R_{\mathrm{had}}^{\ell}=\frac{\Gamma_{\mathrm{had}}}{\Gamma_{\ell}}$
$A_{\mathrm{fb}}^{\circ, \ell}=\frac{3}{4} A_{\mathrm{e}} A_{\ell}$. 

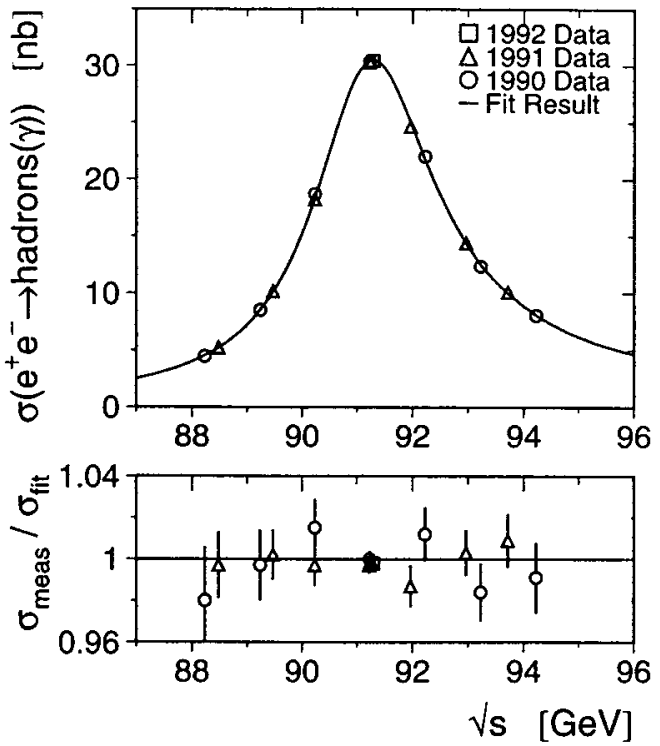

Fig. 13. The cross section, $\sigma_{\text {had }}(s)$, for the process $e^{+} e^{-} \rightarrow$ hadrons $(\gamma)$ as a function of $\sqrt{s}$. Points with error bars are the measured values. The solid line is the result of the fit to the combined cross-section and forwardbackward asymmetry data, assuming lepton universality. Also shown is the ratio of the measured cross section to the fitted cross section for all center-of-mass energy points

Figures 13, 14, 15 and 16 show the measured cross sections compared to the result of the fit assuming lepton universality, as well as the ratio between the measured and fitted cross sections. Figures 17, 18 and 19 show the measured forward-backward asymmetries compared to the result of this fit. The measurement of the average tau polarization, $\mathscr{P}_{\tau}$, adds important additional information about $\bar{g}_{V}^{\ell}$ and $\bar{g}_{A}^{\ell}$, because [23]:

$\mathscr{P}_{\tau}\left(s=m_{\mathrm{Z}}^{2}\right)=-A_{\tau}=-\frac{2 \bar{g}_{V}^{\tau} \bar{g}_{A}^{\tau}}{\left(\bar{g}_{V}^{\tau}\right)^{2}+\left(\bar{g}_{A}^{\tau}\right)^{2}}$

which also determines the relative sign of $\bar{g}_{V}^{\ell}$ and $\bar{g}_{A}^{\ell}$. We determine the leptonic effective coupling constants using the total cross sections and leptonic forward-backward asymmetries, including our measurement of $\mathscr{P}_{\tau}=-0.132 \pm 0.033$ [3]. The results are shown in Table 18. The measurements for the three charged lepton species are in good agreement and confirm the hypothesis of lepton universality. Assuming this hypothesis, we determine the effective coupling constants for charged leptons to be:

$\bar{g}_{V}^{\ell}=-0.0378_{-0.0042}^{+0.0045}$

$\bar{g}_{A}^{\ell}=-0.4998 \pm 0.0014$.

The allowed region of values in the $\left(\bar{g}_{V}^{\ell}, \bar{g}_{A}^{\ell}\right)$ plane is shown in Fig. 20. Good agreement with the predictions of the SM is observed.

From the above values of the effective coupling constants and their correlation, we derive the effective electroweak mixing angle, $\sin ^{2} \bar{\theta}_{W}$, and $\bar{\rho}$ :

$$
\begin{aligned}
\sin ^{2} \bar{\theta}_{W} & =0.2312 \pm 0.0022 \\
\bar{\rho} & =0.9992 \pm 0.0056 .
\end{aligned}
$$
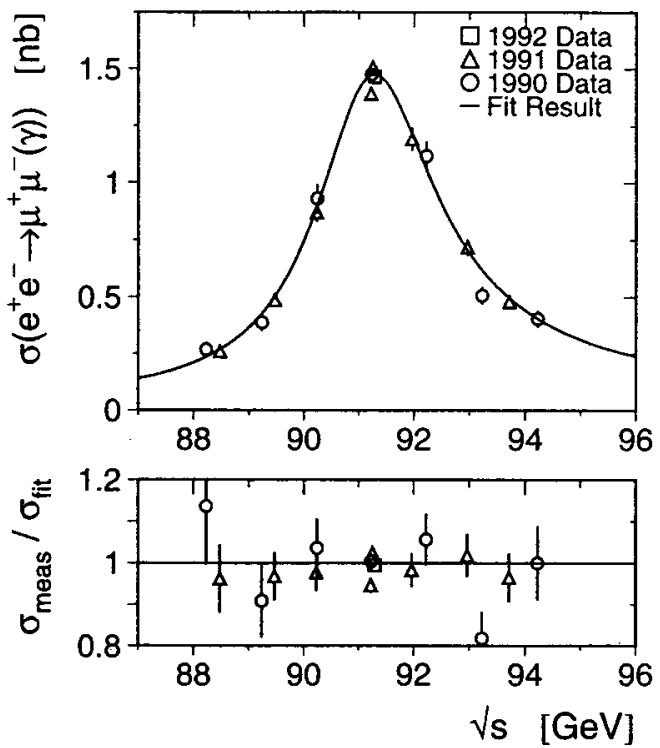

Fig. 14. The cross section, $\sigma_{\mu}(s)$, for the process $e^{+} e^{-} \rightarrow \mu^{+} \mu^{-}(\gamma)$ as a function of $\sqrt{s}$. Points with error bars are the measured values. The solid line is the result of the fit to the combined cross-section and forwardbackward asymmetry data, assuming lepton universality. Also shown is the ratio of the measured cross section to the fitted cross section for all center-of-mass energy points

As in the case of leptons, the $b \bar{b}$ forward-backward asymmetry also gives information about $\sin ^{2} \bar{\theta}_{W}$ [36]. Including our measurement of $A_{\mathrm{fb}}^{b}=0.086 \pm 0.017$ [37], we obtain:

$\sin ^{2} \bar{\theta}_{W}=0.2319 \pm 0.0018$

\subsection{Results in the framework of the standard model}

The measurements of the hadronic cross sections, leptonic cross sections and forward-backward asymmetries, average tau polarization, $b \bar{b}$ forward-backward asymmetry are used to estimate the mass of the top quark in the framework of the SM. We also include our measurement of the $Z \rightarrow b \bar{b}$ partial width, $\Gamma_{\mathrm{b}} / \Gamma_{\text {had }}=0.222 \pm 0.008$ [38]. Using the third approach described above, the free parameters in the fit based on the SM are $m_{\mathrm{Z}}, m_{t}$ and the strong coupling constant, $\alpha_{s}$. The results are:

$$
\begin{aligned}
M_{\mathrm{Z}} & =91.195 \pm 0.009 \mathrm{GeV} \\
\alpha_{\mathrm{s}} & =0.142 \pm 0.013 \pm 0.002 \text { (Higgs) } \\
m_{t} & =142_{-49-19}^{+37+18} \text { (Higgs) GeV. }
\end{aligned}
$$

The second error expresses the shift in the central values of $\alpha_{\mathrm{s}}$ and $m_{t}$ for a variation in the mass of the Higgs boson, $m_{H}$, from 60 to $1000 \mathrm{GeV}$ around the central value of $300 \mathrm{GeV}$. The correlation between $\alpha_{\mathrm{s}}$ and $m_{t}$ is shown in Fig. 21. The measurement of $R_{\text {had }}^{\ell}$ alone also constrains the strong coupling constant [39]. Our measurement of $R_{\mathrm{had}}^{\ell}$ yields $\alpha_{\mathrm{s}}=0.141 \pm 0.012$. Both $\alpha_{\mathrm{s}}$ values are in agreement with our measurement of the strong coupling constant from hadronic event topologies and tau decays, $\alpha_{\mathrm{s}}=0.123 \pm 0.006$ [4]. Constraining $\alpha_{s}$ to this independent result, the determination of $m_{t}$ improves: 
Table 18. Results on the effective coupling constants, $\bar{g}_{V}^{\ell}$ and $\bar{g}_{A}^{\ell}$, derived from the tau-polarization, forward-backward asymmetry and cross-section data. SM expectations are listed using the fitted $Z$ mass, $m_{Z}=91.195 \pm 0.009 \mathrm{GeV}, \alpha_{\mathrm{s}}=0.123 \pm 0.006$ and taking $m_{t}=150 \pm 50 \mathrm{GeV}, m_{H}=300_{-240}^{+700} \mathrm{GeV}$

\begin{tabular}{|c|c|c|c|}
\hline Parameter & \multicolumn{2}{|c|}{ Treatment of charged leptons } & $\begin{array}{c}\text { Standard } \\
\text { Model }\end{array}$ \\
\hline$m_{Z}[\mathrm{MeV}]$ & $91195 \pm 6 \pm 7$ (LEP) & $91195 \pm 6 \pm 7$ (LEP) & - \\
$\Gamma_{Z}[\mathrm{MeV}]$ & $2494 \pm 9 \pm 5$ (LEP) & $2495 \pm 9 \pm 5$ (LEP) & $2491_{-18}^{+21}$ \\
$\Gamma_{\mathrm{had}}[\mathrm{MeV}]$ & $1749 \pm 11$ & $1748 \pm 10$ & $1739_{-14}^{+16}$ \\
\hline $\bar{g}_{V}^{e}$ & $-0.0364_{-0.0082}^{+0.0096}$ & - & \\
$\bar{g}_{V}^{\mu}$ & $-0.0402_{-0.0211}^{+0.0153}$ & - & \\
$\bar{g}_{Y}^{\tau}$ & $-0.0384 \pm 0.0078$ & - & \\
$\bar{g}_{V}^{\tau}$ & - & $-0.0378_{-0.0042}^{+0.0045}$ & $-0.0342_{-0.0057}^{+0.0042}$ \\
\hline $\bar{g}_{A}^{e}$ & $-0.4998 \pm 0.0016$ & - & \\
$\bar{g}_{A}^{e}$ & $-0.4987_{-0.0030}^{+0.0026}$ & - & \\
$\bar{g}_{A}^{\tau}$ & $-0.5014 \pm 0.0029$ & - & \\
$\bar{g}_{A}^{\ell}$ & - & $-0.4998 \pm 0.0014$ & $-0.5007_{-0.0014}^{+0.0012}$ \\
\hline$\chi^{2} /$ DOF & $86 / 104$ & $87 / 108$ & - \\
\hline
\end{tabular}
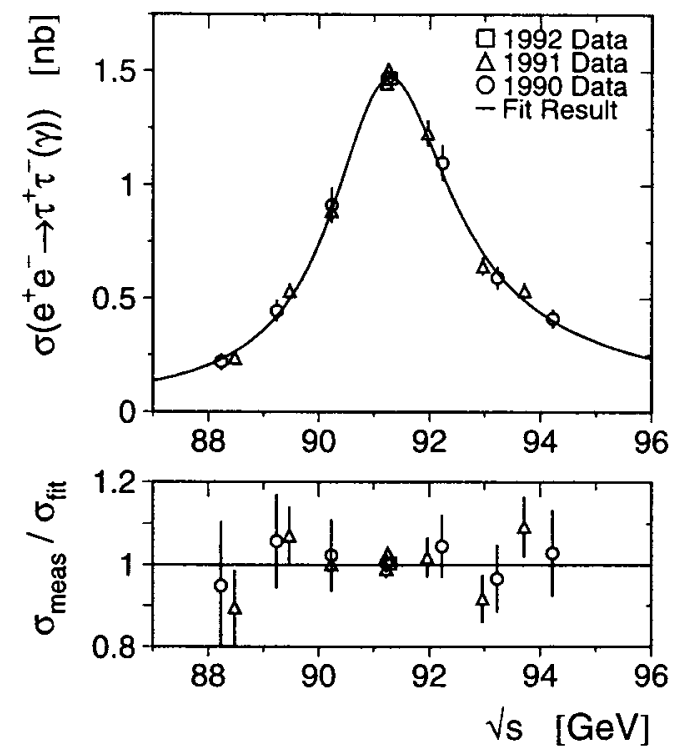

Fig. 15. The cross section, $\sigma_{\tau}(s)$, for the process $e^{+} e^{-} \rightarrow \tau^{+} \tau^{-}(\gamma)$ as a function of $\sqrt{s}$. Points with error bars are the measured values. The solid line is the result of the fit to the combined cross-section and forwardbackward asymmetry data, assuming lepton universality. Also shown is the ratio of the measured cross section to the fitted cross section for all center-of-mass energy points

$m_{t}=158_{-40}^{+32} \pm 19$ (Higgs) $\mathrm{GeV}$,

while the result on $m_{Z}$ is unchanged. The result on $m_{t}$ is consistent with current limits on the top-quark mass derived from direct searches [40].

From the fitted values of $m_{\mathrm{Z}}$ and $m_{t}$, and for $\alpha_{\mathrm{s}}=$ $0.123 \pm 0.006$ the following quantities are derived:

$$
\begin{aligned}
\sin ^{2} \bar{\theta}_{W} & =0.2326 \pm 0.0012 \\
\sin ^{2} \theta_{W} & =0.2260 \pm 0.0042 \\
\Delta r & =0.045 \pm 0.013 \\
m_{W} & =80.22 \pm 0.22 \mathrm{GeV}
\end{aligned}
$$

where $\sin ^{2} \theta_{W}$ is defined as $\left(1-m_{W}^{2} / m_{Z}^{2}\right)$. The error on these results due to the uncertainty in the mass of the Higgs boson is negligible. The value of the mass of the $\mathrm{W}$ bo-
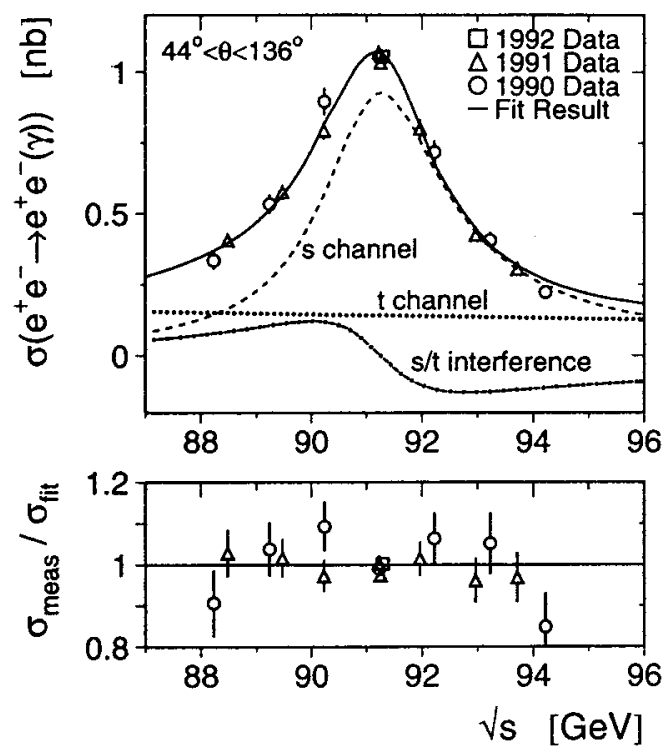

Fig. 16. The cross section, $\sigma_{\mathrm{e}}(s)$, for the process $e^{+} e^{-} \rightarrow e^{+} e^{-}(\gamma)$ as a function of $\sqrt{s}$. Points with error bars are the measured values. The solid line is the result of the fit to the combined cross-section and forwardbackward asymmetry data, assuming lepton universality. The lines showing the contributions of the $s$ channel, $t$ channel and $s / t$ interference are computed with the ALIBABA program. Also shown is the ratio of the measured cross section to the fitted cross section for all center-of-mass energy points

son as obtained above is in good agreement with the direct measurements of $m_{W}$ [41].

In order to disentangle new physics beyond the SM from the possibly large, unknown top-quark corrections of leading order $G_{F} m_{t}^{2}$, four new parameters, $\epsilon_{1}, \epsilon_{2}, \epsilon_{3}$ and $\epsilon_{\mathrm{b}}$, have been introduced [42]. Their merit lies in separating out the $m_{t}$ dependent effects in $\epsilon_{1}$ and $\epsilon_{\mathrm{b}}$, and other $\left(m_{H}\right)$ effects in $\epsilon_{2}$ and $\epsilon_{3}$. The LEP data at the $Z$ resonance constrain $\epsilon_{1}$, $\epsilon_{3}$ and $\epsilon_{b}$, the latter by the measurements of $b$ quarks. Our measurements of the hadronic cross sections and leptonic cross sections and asymmetries constrain the parameters $\epsilon_{1}$ and $\epsilon_{3}$, which are defined as:

$$
\begin{aligned}
& \epsilon_{1}=\Delta \bar{\rho} \\
& \epsilon_{3}=\left(1-s_{0}^{2}\right) \Delta \bar{\rho}+\left(1-2 s_{0}^{2}\right)\left(\sin ^{2} \bar{\theta}_{W} / s_{0}^{2}-1\right)
\end{aligned}
$$




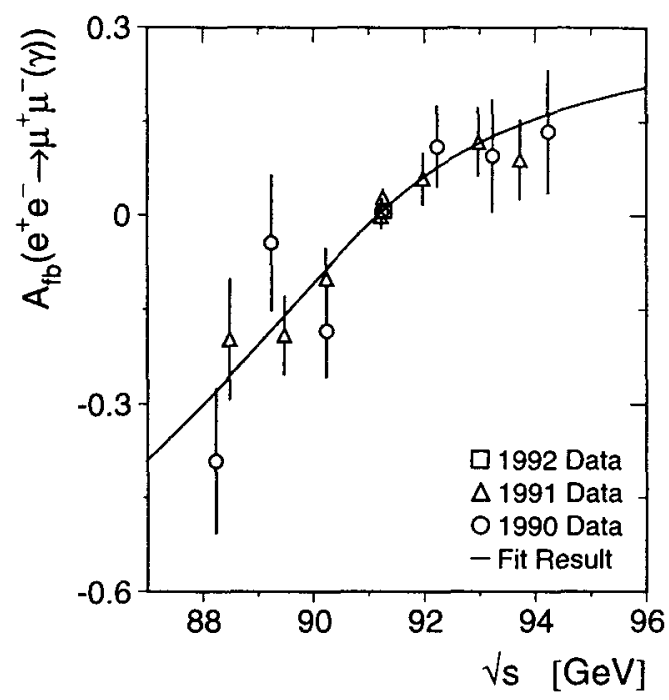

Fig. 17. The forward-backward asymmetry, $\mathrm{A}_{\mathrm{fb}}^{\mu}(\mathrm{s})$, for the process $e^{+} e^{-} \rightarrow$ $\mu^{+} \mu^{-}(\gamma)$ as a function of $\sqrt{s}$. Points with error bars are the measured values. The solid curve is the result of the fit to the combined cross-section and forward-backward asymmetry data, assuming lepton universality

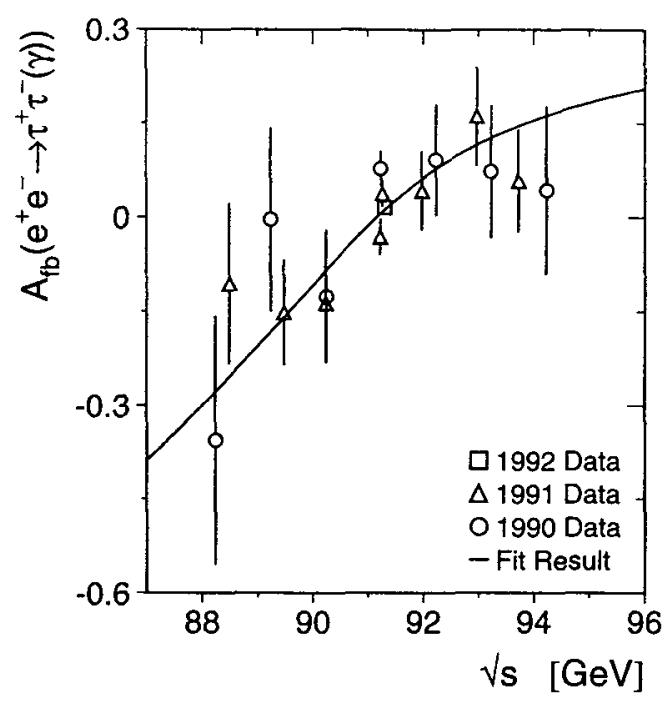

Fig. 18. The forward-backward asymmetry, $\mathrm{A}_{\mathrm{fb}}^{\tau}(\mathrm{s})$, for the process $e^{+} e^{-} \rightarrow$ $\tau^{+} \tau^{-}(\gamma)$ as a function of $\sqrt{s}$. Points with error bars are the measured values. The solid curve is the result of the fit to the combined cross-section and forward-backward asymmetry data, assuming lepton universality

$s_{0}^{2}\left(1-s_{0}^{2}\right)=\left[\pi \alpha\left(m_{z}^{2}\right)\right] /\left[\sqrt{2} \mathrm{G}_{\mathrm{F}} \mathrm{m}_{\mathrm{Z}}^{2}\right]$

We find the following values for $\epsilon_{1}$ and $\epsilon_{3}$ :

$\epsilon_{1}=-0.0008 \pm 0.0056$

$\epsilon_{3}=-0.0012 \pm 0.0073$.

The range of allowed values in the $\left(\epsilon_{1}, \epsilon_{3}\right)$ plane is shown in Fig. 22. Our values of the parameters $\epsilon_{1}$ and $\epsilon_{3}$ are consistent with the predictions of the SM.

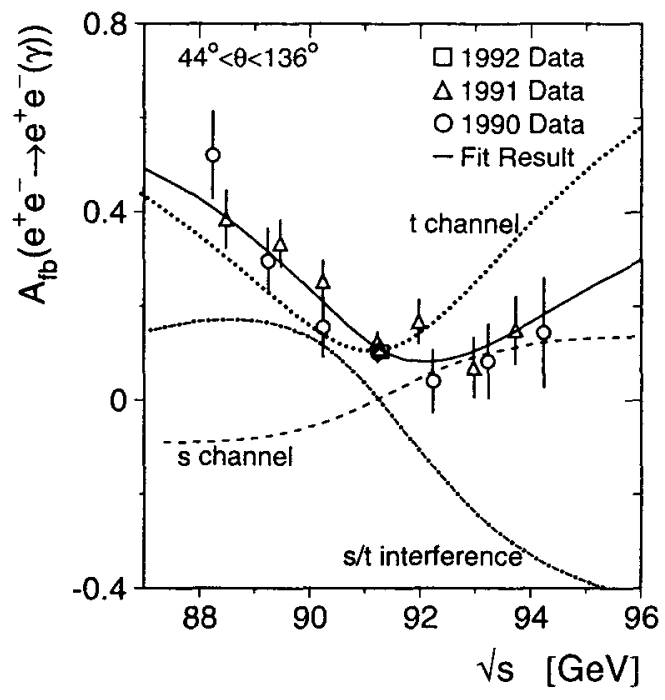

Fig. 19. The forward-backward asymmetry, $A_{\mathrm{fb}}^{\mathrm{e}}(\mathrm{s})$, for the process $e^{+} e^{-} \rightarrow$ $e^{+} e^{-}(\gamma)$ as a function of $\sqrt{s}$. Points with error bars are the measured values. The solid curve is the result of the fit to the combined cross-section and forward-backward asymmetry data, assuming lepton universality. The lines showing the contributions of the $s$ channel, $\sigma_{\mathrm{f}}^{s}-\sigma_{\mathrm{b}}^{\mathrm{s}}, \mathrm{t}$ channel, $\sigma_{\mathrm{f}}^{\mathrm{t}}-\sigma_{\mathrm{b}}^{\mathrm{t}}$, and $s / t$ interference, $\sigma_{\mathrm{f}}^{s / t}-\sigma_{b}^{s / t}$, normalized to the total cross section, $\sigma^{s}+\sigma^{t}+\sigma^{s / t}$, are computed with the ALIBABA program

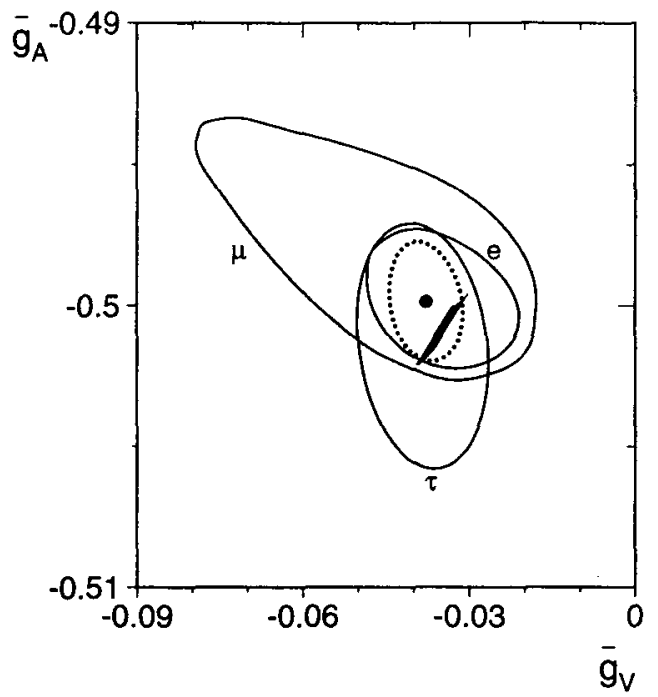

Fig. 20. Correlation between the effective coupling constants of the neutral weak current, $\bar{g}_{V}$ and $\bar{g}_{A}$, for charged leptons. The $68 \%$ confidence level contours for the two parameters are shown, for each lepton species separately (solid contours), and under the assumption of lepton universality (dotted contour). The band shows the prediction according to the SM, using the fitted $Z$ mass, $m_{Z}=91.195 \pm 0.009 \mathrm{GeV}, \alpha_{s}=0.123 \pm 0.006$ and taking $m_{t}=150 \pm 50 \mathrm{GeV}, m_{H}=300_{-240}^{+700} \mathrm{GeV}$

\section{Summary and conclusion}

We determine the electroweak parameters from the measurements of the reactions $e^{+} e^{-} \rightarrow$ hadrons $(\gamma), e^{+} e^{-} \rightarrow$ $\mu^{+} \mu^{-}(\gamma), e^{+} e^{-} \rightarrow \tau^{+} \tau^{-}(\gamma)$ and $e^{+} e^{-} \rightarrow e^{+} e^{-}(\gamma)$. This includes the data collected by the L3 detector in the year 1992 , when LEP was running exclusively at the center-of-mass en- 


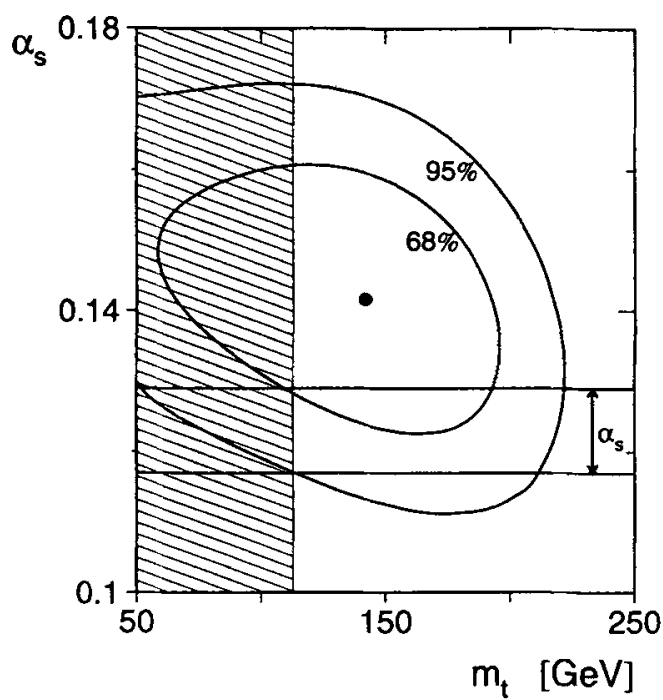

Fig. 21. Correlation between the strong coupling constant, $\alpha_{s}$, and the mass of the top quark, $m_{\mathrm{t}}$, with $m_{\mathrm{H}}$ fixed to $300 \mathrm{GeV}$. The $68 \%$ and $95 \%$ confidence level contours for the two parameters are shown, together with our measurement of $\alpha_{\mathrm{s}}$ from hadronic event topologies and tau decays $\left(\alpha_{s}=0.123 \pm 0.006\right)$, and the limit on $m_{\mathrm{t}}$ from direct searches $\left(m_{\mathrm{t}}>\right.$ $113 \mathrm{GeV})$

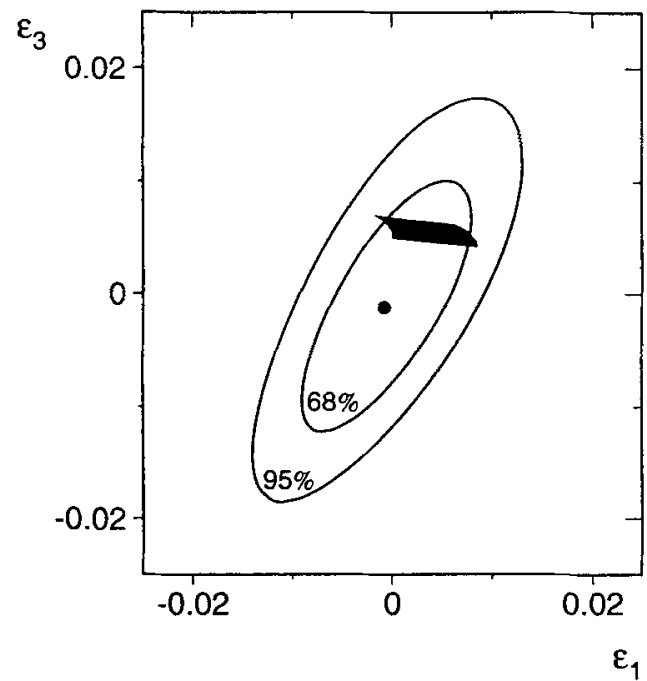

Fig. 22. Correlation between the parameters $\epsilon_{1}$ and $\epsilon_{3}$ (see text). The $68 \%$ and $95 \%$ confidence level contours for the two parameters are shown. The band shows the prediction according to the $\mathrm{SM}$, using the fitted $Z$ mass, $m_{Z}=91.195 \pm 0.009 \mathrm{GeV}, \alpha_{s}=0.123 \pm 0.006$ and taking $m_{t}=150 \pm$ $50 \mathrm{GeV}, m_{H}=300_{-240}^{+700} \mathrm{GeV}$

ergy of $\sqrt{s}=91.29 \mathrm{GeV}$. Together with the data collected in 1990 and 1991 in the energy range $88<\sqrt{s}<95 \mathrm{GeV}$, the total data sample used for this analysis corresponds to $1.09 \cdot 10^{6}$ hadronic and $0.98 \cdot 10^{5}$ leptonic $Z$ decays selected.

All our measurements support the hypothesis of lepton universality. From the hadronic and leptonic cross-section data, we determine the properties of the $Z$ boson:

$$
\begin{aligned}
M_{\mathrm{Z}} & =91195 \pm 9 \mathrm{MeV} \\
\Gamma_{\mathrm{Z}} & =2494 \pm 10 \mathrm{MeV}
\end{aligned}
$$

$$
\begin{aligned}
\Gamma_{\mathrm{had}} & =1748 \pm 10 \mathrm{MeV} \\
\Gamma_{\ell} & =83.49 \pm 0.46 \mathrm{MeV} .
\end{aligned}
$$

The corresponding invisible width of $496.5 \pm 7.9 \mathrm{MeV}$ constrains, within the SM, the number of light neutrino species to be:

$N_{\nu}=2.981 \pm 0.050$.

Including the leptonic forward-backward asymmetries and the average tau polarization, the effective neutral weak current coupling constants for charged leptons are given by:

$\bar{g}_{V}^{\ell}=-0.0378_{-0.0042}^{+0.0045}$

$\bar{g}_{A}^{\ell}=-0.4998 \pm 0.0014$.

Within the SM, and including our measurement of the $\mathrm{Z} \rightarrow$ $\mathrm{b} \bar{b}$ forward-backward asymmetry and partial decay width, we determine:

$$
\begin{aligned}
\alpha_{\mathrm{s}} & =0.142 \pm 0.013 \pm 0.002 \text { (Higgs) } \\
m_{t} & =142_{-49-19}^{+37+18} \text { (Higgs) GeV. }
\end{aligned}
$$

This value of $\alpha_{\mathrm{s}}$ is in agreement with our measurement of $\alpha_{\mathrm{s}}$ from hadronic event topologies and tau decays, $\alpha_{s}=0.123 \pm$ 0.006 . Constraining $\alpha_{s}$ to this independent measurement, we derive:

$m_{t}=158_{-40}^{+32} \pm 19$ (Higgs) GeV.

Alternatively, we determine a value for the mass of the $\mathrm{W}$ boson:

$m_{W}=80.22 \pm 0.22 \mathrm{GeV}$,

or, expressed in terms of the weak mixing angle:

$\sin ^{2} \theta_{W}=1-\frac{m_{W}^{2}}{m_{\mathrm{Z}}^{2}}=0.2260 \pm 0.0042$,

which corresponds to the following value of the effective electroweak mixing angle:

$\sin ^{2} \bar{\theta}_{W}=0.2326 \pm 0.0012$.

All our measurements are in good agreement with the predictions of the SM.

Acknowledgement. We wish to express our gratitude to the CERN accelerator divisions for the excellent performance of the LEP machine. We acknowledge the contributions of all the engineers and technicians who have participated in the construction and maintenance of this experiment.

\section{References}

1. S.L. Glashow: Nucl. Phys. 22 (1961) 579; S. Weinberg: Phys. Rev. Lett. 19 (1967) 1264; A. Salam: Elementary particle theory, N. Svarthiolm (ed.) p 367. Stockholm: Almquist and Wiksell 1968.

2. M. Gell-Mann: Acta Phys. Aust., Suppl. IX (1972) 733; H. Fritzsch, M. Gell-Mann: 16th International Conference on High Energy Physics, Batavia, 1972, J.D. Jackson, A. Roberts (eds.) National Accelerator Laboratory (1972); H. Fritsch, M. Gell-Mann, H. Leutwyler, Phys. Lett. B 47 (1973) 365; D.J. Gross, F. Wilczek: Phys. Rev. Lett. 30 (1973) 1343; D.J. Gross, F. Wilczek: Phys. Rev. D 8 (1973) 3633 H.D. Politzer: Phys. Rev. Lett. 30 (1973) 1346; G. 't Hooft: Nucl Phys. B 33 (1971) 173

3. L3 Coll., B. Adeva et al.: Z. Phys. C 51 (1991) 179

4. L3 Coll., O. Adriani et al.: Phys. Reports 236 (1993) 
5. ALEPH Coll., D. Decamp et al.: Z. Phys. C 53 (1992) 1; ALEPH Coll., D. Buskulic et al.: Z. Phys. C. 59 (1993) 369; ALEPH Coll., D. Buskulic et al.: CERN-PPE/94-30; DELPHI Coll., P. Abreu et al.: Nucl. Phys. B 367 (1991) 511; DELPHI Coll., P. Abreu et al.: CERNPPE/94-08; DELPHI Coll., P. Abreu et al.: CERN/PPE/94-31; OPAL Coll., G. Alexander et al.: Z. Phys. C 52 (1991) 175; OPAL Coll. P.D. Acton et al.: Z. Phys. C 58 (1993) 219; OPAL Coll., R. Akers et al.: CERN-PE/93-146

6. L3 Coll., B. Adeva et al.: Nucl. Instrum. Methods A 289 (1990) 35

7. R. Brun et al.: GEANT 3, CERN DD/EE/84-1 (Revised) 1987

8. H. Fesefeldt: RWTH Aachen Preprint PITHA 85/02 (1985)

9. S. Jadach, B.F.L. Ward: Phys. Rev. D 40 (1989) 3582

10. S. Jadach et al.: Phys. Lett. B 268 (1991) 253; S. Jadach et al.: Comp. Phys. Commun. 70 (1992) 305

11. M. Böhm, A. Denner, W. Hollik: Nucl. Phys. B 304 (1988) 687; F.A. Berends, R. Kleiss, W. Hollik: Nucl. Phys. B 304 (1988) 712

12. F.A. Berends, R. Kleiss: Nucl. Phys. B 186 (1981) 22

13. W. Beenakker, B. Pietrzyk: Preprint CERN-TH.6760/92, CERN, 1992

14. O. Adriani et al.: Nucl. Instrum. Methods A 302 (1991) 53

15. T. Sjöstrand: Comp. Phys. Commun. 39 (1986) 347; T. Sjöstrand, B. Bengtsson: Comp. Phys. Comm. 43 (1987) 367

16. G. Marchesini, B. Webber: Nucl. Phys. B 310 (1988) 461; G. Marchesini et al.: Comp. Phys. Commun. 67 (1992) 465

17. S. Jadach, B.F.L. Ward, Z. Wạs: Com. Phys. Commun. 66 (1991) 276

18. F.A. Berends, P.H, Daverveldt, R. Kleiss: Nucl. Phys. B 253 (1985) 441

19. D. Bardin et al.: FORTRAN package ZFITTER, and preprint CERNTH.6443/92; D. Bardin et al.: Z. Phys. C 44 (1989) 493; D. Bardin et al.: Nucl. Phys. B 351 (1991) 1; D. Bardin et al.: Phys. Lett. B 255 (1991) 290; J. Fleischer et al.: Phys. Lett. B 293 (1992) 437; S. Jadach et al.: Phys. Lett. B 280 (1992) 129; B. Kniehl et al.: Phys. Lett. B 209 (1988) 337; R. Barbieri et al.: CERN-TH-6713-92

20. J.H. Field: Preprint UGVA-DPNC 1993/9-160, University of Geneva, 1993, to be published in Phys. Lett. B

21. W. Beenakker, F.B. Berends, S.C. van der Marck: Nucl. Phys. B 349 (1991) 323

22. The working group on LEP energy, $\mathrm{L}$. Arnaudon et al.: Preprint CERNPPE/92-125, CERN, 1992; Preprint CERN-SL/93-21 (DI), CERN, 1993
23. L3 Coll., O. Adriani et al.: Phys. Lett. B 315 (1993) 494

24. LEP Collaborations: Phys. Lett. B 307 (1993) 187

25. LEP Collaborations: ALEPH, DELPHI, L3, OPAL and the LEP Electroweak Working Group: Updated parameters of the $Z$ resonance from combined preliminary data of the LEP experiments. Preprint CERNPPE/93-157, CERN, 1993

26. F.A. Berends et al., in: $Z$ Physics at LEP 1, CERN Report CERN 89-08 (1989), G. Altarelli, R. Kleiss, C. Verzegnassi (eds.), vol. 1, p. 89

27. M. Böhm et al., in: $Z$ Physics at LEP 1, CERN Report CERN 89-08 (1989) G. Altarelli, R. Kleiss, C. Verzegnassi (eds.), vol. 1, p. 203

28. M. Consoli et al., in: $Z$ Physics at LEP 1, CERN Report CERN 89-08 (1989) G. Altarelli, R. Kleiss, C. Verzegnassi (eds.), vol. 1, p. 7

29. G. Burgers et al., in: Z Physics at LEP 1, CERN Report CERN 89-08 (1989) G. Altarelli, R. Kleiss, C. Verzegnassi (eds.), vol. 1, p. 55

30. F. James: CERN Program Library Long Writeup D506 MINUIT, CERN, 1993

31. G. Isidori: Phys. Lett. B 314 (1993) 139

32. M. Grünewald, S. Kirsch: Preprint CERN-PPE/93-188, CERN, 1993

33. L3 Coll., B. Adeva et al.: Phys. Let. B 275 (1992) 209; L3 Coll., O. Adriani et al.: Phys. Lett. B 292 (1992) 463; OPAL Coll., M.Z. Akrawy et al.: Z. Phys. C 50 (1991) 373; ALEPH Coll., D. Buskulic et al.: Phys. Lett. B 313 (1993) 520

34. S. Jadach et al,, in: Z Physics at LEP 1, CERN Report CERN 89-08 (1989) G. Altarelli, R. Kleiss, C. Verzegnassi (eds.), vol. 1, p. 235

35. L3 Coll., O. Adriani et al.: Phys. Lett. B 294 (1992) 466

36. J. Kühn et al., in: Z Physics at LEP 1, CERN Report CERN 89-08 (1989) G. Altarelli, R. Kleiss, C. Verzegnassi (eds.), vol. 1, p. 267

37. L3 Coll., O. Adriani et al.: Phys. Lett. B 292 (1992) 454

38. L3 Coll., O. Adriani et al.: Phys. Lett. B 307 (1993) 237

39. G. Passarino: Phys. Lett. B 313 (1993) 213

40. P. Tipton: The top search at CDF; N. Hadley: The top search at D0; Talks presented at the 1993 International Symposium on Lepton-Photon Interactions at High Energies, Cornell, to appear in the proceedings

41. CDF Coll., F. Abe et al.: Phys. Rev. Lett. 65 (1990) 2243; CDF Coll., F. Abe et al.: Phys. Rev. D 43 (1991) 2070; UA2 Coll., J. Alitti et al.: Phys. Lett. B 276 (1992) 354

42. G. Altarelli, R. Barbieri: Phys. Lett. B 253 (1990) 161; G. Altarelli, R. Barbieri, S. Jadach: Nucl. Phys. B 369 (1993) 3; G. Altarelli, R. Barbieri, F. Caravaglios: Preprint CERN-TH.6770/93, CERN, 1993 